\title{
Symmetries and Collective Excitations in Large Superconducting Circuits
}

\author{
David G. Ferguson, ${ }^{1}$ A. A. Houck, ${ }^{2}$ and Jens Koch $^{1}$ \\ ${ }^{1}$ Department of Physics and Astronomy, Northwestern University, Evanston, Illinois 60208, USA \\ ${ }^{2}$ Department of Electrical Engineering, Princeton University, Princeton, New Jersey 08544, USA \\ (Received 4 October 2012; revised manuscript received 6 December 2012; published 17 January 2013) \\ In this work, we present theoretical tools suitable for quantitative modeling of large superconducting \\ circuits that include one-dimensional Josephson-junction arrays. The large number of low-energy degrees \\ of freedom and the peculiar interactions between them induced by flux quantization present a considerable \\ challenge to the detailed modeling of such circuits. For the concrete example of the fluxonium device, we \\ show how to address this challenge. Starting from the complete degrees of freedom of the circuit, we \\ employ the relevant collective modes and circuit symmetries to obtain a systematic approximation \\ scheme. Important circuit symmetries include approximate invariance under the symmetric group and \\ lead to considerable simplifications of the theory. Selection rules restrict the possible coupling among \\ different collective modes and help explain the remarkable accuracy of previous simplified models. Using \\ this strategy, we obtain new predictions for the energy spectrum of the fluxonium device that can be tested \\ with current experimental technology.
}

DOI: 10.1103/PhysRevX.3.011003

Subject Areas: Mesoscopics, Quantum Physics, Superconductivity

\section{INTRODUCTION}

In the search for a viable architecture for solid-state quantum-information processing, superconducting circuits have been the focus of immense interest [1-4]. While research efforts have led to the remarkable improvement of coherence times by nearly 5 orders of magnitude [5] relative to those in the pioneering experiments a decade ago [6,7], superconducting circuits have remained extremely simple-especially when compared to circuits found in commonplace electronic devices. Most superconducting quantum circuits such as phase, flux, or charge qubits consist of less than a handful of circuit elements.

Experiments with the fluxonium device-a superconducting circuit with more than 40 Josephson junctionshave shown that a larger number of Josephson junctions, and hence degrees of freedom, is not necessarily penalized by reduced coherence times [8,9]. Experimental studies of linear Josephson-junction arrays have advanced at a rapid pace [10-16]. However, despite considerable theoretical work [17-27], methods for detailed modeling of larger circuit networks are needed to successfully chart the future territory of quantum coherence in networks of increasing size to, e.g., further explore the possibility of topological protection from decoherence [28-31]. The description presents a considerable challenge to theory due to the combination of several factors: the nonlinearity induced by Josephson junctions, the increased number of lowenergy degrees of freedom, and the peculiar interactions between them induced by flux quantization. As a key step

Published by the American Physical Society under the terms of the Creative Commons Attribution 3.0 License. Further distribution of this work must maintain attribution to the author(s) and the published article's title, journal citation, and DOI. for mastering these difficulties, we present theory for the fluxonium device that starts from the complete circuit degrees of freedom. We demonstrate that circuit symmetries play a crucial role in the organization of the excitation spectrum and, employing the relevant collective modes and their approximate decoupling [26], we obtain a systematic approximation scheme.

Nonlinearity, interactions, and a large number of degrees of freedom are challenges commonly encountered in the study of many-electron atoms. Our symmetry-based approach resembles methods familiar from atomic and molecular physics where the weak breaking of symmetries leads to the well-known lifting of degeneracies in the fine and hyperfine structures of spectra [32]. For the fluxonium circuit, we demonstrate that approximate symmetry under the unitary group and under permutations of junction variables divides the excitation spectrum into nearly degenerate subspaces. For realistic parameters, the careful study of perturbations allows us to refine our description and provide new predictions for the collective excitations of the circuit.

\section{FLUXONIUM CIRCUIT}

The fluxonium device [8] (Fig. 1) consists of a Josephson-junction array with a large number $N \gg 1$ of nominally identical tunneling junctions. One additional smaller junction (the "black sheep") shunts the array. The superconducting loop formed in this manner can be biased with an external magnetic flux $\Phi_{\text {ext }}$, making the energy spectrum tunable. As is typical of superconducting circuits, the nature of eigenstates and their detailed energy spectrum are governed by the competition between charge transfers across junctions due to Cooper-pair tunneling and charging effects due to excess electric charge on individual 


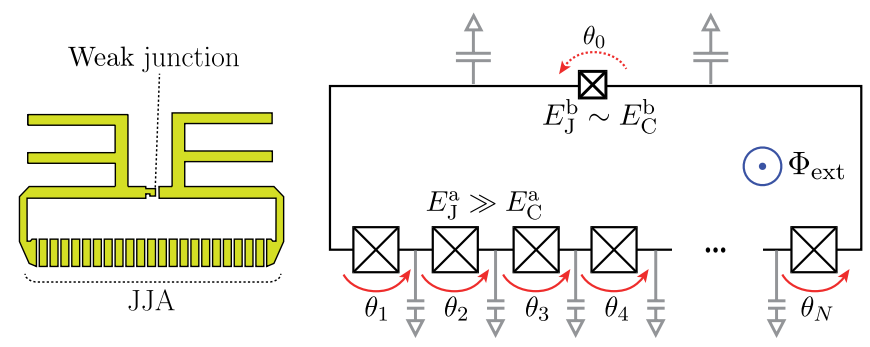

FIG. 1. Circuit of the fluxonium device: a Josephson-junction array (JJA) of nominally identical junctions with Josephson energy $E_{\mathrm{J}}^{\mathrm{a}}$ and charging energy $E_{\mathrm{C}}^{\mathrm{a}}$, shunting one weaker junction (Josephson and charging energy $E_{\mathrm{J}}^{\mathrm{b}}$ and $E_{\mathrm{C}}^{\mathrm{b}}$, respectively).

islands. While charge transfers favor charge delocalization and definite phase differences across each junction, charging effects promote charge localization with definite Cooper-pair numbers on each superconducting island. The ratio of Josephson energy $\left(E_{\mathrm{J}}\right)$ to charging energy $\left(E_{\mathrm{C}}\right)$ of the involved junctions quantifies this interplay. We use the superscripts " $a$ " and "b" in this article to distinguish between array $\left(E_{\mathrm{J}}^{\mathrm{a}} / E_{\mathrm{C}}^{\mathrm{a}} \gg 1\right)$ and black-sheep parameters $\left(E_{\mathrm{J}}^{\mathrm{b}} \sim E_{\mathrm{C}}^{\mathrm{b}}\right)$.

The energy spectrum and corresponding eigenstates of the superconducting circuit $\mathcal{C}$ are governed by the stationary Schrödinger equation $H_{\mathcal{C}}|\psi\rangle=E|\psi\rangle$, in which the circuit Hamiltonian is obtained from the Lagrangian $\mathcal{L}=T-U$ by circuit quantization $[33,34]$. For each junction, Josephson tunneling produces a potential-energy term $U_{m}=-E_{\mathrm{J} m} \cos \theta_{m}$, where $\theta_{m}$ denotes the phase difference across junction $m$. The dominant kinetic-energy contributions arise from the charging of junction capacitances, $T_{m}=\frac{1}{2} C_{\mathrm{J} m} V_{\mathrm{J} m}^{2}$. Here, the voltage drop across junction $m$ is linked to $\dot{\theta}_{m}$ via Josephson's phase-evolution equation $\dot{\theta}_{m}=2 \pi V_{\mathrm{J} m} / \Phi_{0}$, and charging energies are related to capacitances via $E_{\mathrm{C}}=e^{2} / 2 C$.

The requirement for the superconducting phase to be single valued (modulo integer multiples of $2 \pi$ ) leads to fluxoid quantization [35-37]. It manifests itself as the rigid constraint $\sum_{m=0}^{N} \theta_{m}+\varphi_{\mathrm{ext}}=2 \pi z$, where $z$ is an integer, $\Phi_{0}=h / 2 e$ the superconducting flux quantum, and $\varphi_{\mathrm{ext}}=$ $2 \pi \Phi_{\text {ext }} / \Phi_{0}$ the phase offset due to external magnetic flux. The constraint reduces the number of independent coordinates by one and induces coupling among the remaining junction phases. To incorporate the constraint while maintaining symmetry among array junctions, we eliminate the black-sheep variable $\theta_{0}$ and obtain the Lagrangian

$$
\begin{aligned}
\mathcal{L}= & \frac{\hbar^{2}}{16 E_{\mathrm{C}}^{\mathrm{a}}} \sum_{m} \dot{\theta}_{m}^{2}+\frac{\hbar^{2}}{16 E_{\mathrm{C}}^{\mathrm{b}}}\left[\sum_{m} \dot{\theta}_{m}\right]^{2}+\frac{1}{2} \sum_{m n} \mathcal{G}_{m n} \dot{\theta}_{m} \dot{\theta}_{n} \\
& -\sum_{m} E_{\mathrm{J} m}^{\mathrm{a}} \cos \theta_{m}-E_{\mathrm{J}}^{\mathrm{b}} \cos \left(\sum_{m} \theta_{m}+\varphi_{\mathrm{ext}}\right),
\end{aligned}
$$

where, as a convention, sums over Latin indices always run over the range $1, \ldots, N$. The capacitive term involving the matrix $G$ describes the effects from capacitances between superconducting islands and ground (see Appendix D).

To illustrate the content of Eq. (1), it is instructive to note that $\mathcal{L}$ describes a single fictitious particle inside a periodic potential, albeit in $N$-dimensional space with $N \gg 1$. Alternatively, it can be interpreted as a description of $N$ distinguishable particles, each moving in a 1D periodic potential but subject to a peculiar interaction of collective type induced by flux quantization.

The central idea of our approach in this article is to harness the large amount of symmetry present in the dominant terms of Eq. (1) [38]. In particular, if ground capacitances are negligible and if all array junctions possess the same charging energy $E_{\mathrm{C}}^{\mathrm{a}}$ and Josephson energy $E_{\mathrm{J}, m}^{\mathrm{a}}=E_{\mathrm{J}}^{\mathrm{a}}$, then $\mathcal{L}$ is manifestly $\mathrm{S}_{N}$ symmetric. In other words, any permutation $\sigma \in \mathrm{S}_{N}$ of the array variables, such as

$$
\sigma_{12}\left[\left(\theta_{1}, \theta_{2}, \theta_{3}, \ldots, \theta_{N}\right)\right]=\left(\theta_{2}, \theta_{1}, \theta_{3}, \ldots, \theta_{N}\right),
$$

leaves the Lagrangian invariant for any value of the external flux. We will refer to this idealization as the symmetric-fluxonium model (SFM).

In nonrelativistic quantum mechanics, such discrete symmetries generally lead to degeneracies that are governed by the irreducible representations of the symmetry group. The simplest irreducible representations of the symmetric group $\mathrm{S}_{N}$ are the trivial and alternating representations that are familiar from particle and many-body physics. In those contexts, they dictate the symmetry of wave functions for indistinguishable bosons and fermions. In the case of superconducting circuits, degrees of freedom referring to different junctions generally remain distinguishable, and the full plethora of irreducible representations of $S_{N}$ is realized. In this sense, the SFM constitutes an intriguing example of a many-body system with degenerate eigenstates that obey novel permutation symmetries beyond those of bosons and fermions.

\section{III. $\mathrm{S}_{N}$ SYMMETRIC-FLUXONIUM MODEL}

From the circuit Lagrangian (1), we now extract the relevant collective modes [26] governing the low-energy physics and discuss their connection with effective models employed in previous works $[8,39]$. A key ingredient in the construction of the low-energy modes is the observation that array junctions in fluxonium are dominated by Josephson tunneling, $E_{\mathrm{C}}^{\mathrm{a}} / E_{\mathrm{J}}^{\mathrm{a}} \ll 1$, while the black-sheep parameters $E_{\mathrm{C}}^{\mathrm{b}} \sim E_{\mathrm{J}}^{\mathrm{b}}$ are both roughly of the same order as the array charging energy.

For large arrays with junction number $N \gg E_{\mathrm{J}}^{\mathrm{a}} / E_{\mathrm{J}}^{\mathrm{b}}$, the potential energy $U(\vec{\theta})$ exhibits deep minima at positions where all array coordinates have the identical value

$$
\theta_{m} \simeq-\frac{\bar{\varphi}_{\mathrm{ext}}-2 \pi z}{N+E_{\mathrm{J}}^{\mathrm{a}} / E_{\mathrm{J}}^{\mathrm{b}}}
$$

with the integer $z$ satisfying $|z| \ll N$ and $\bar{\varphi}_{\text {ext }}=$ $\varphi_{\text {ext }} \bmod 2 \pi$. The minima of $U(\vec{\theta})$ are surrounded by large 
energy barriers of height $\geq 2 E_{\mathrm{J}}^{\mathrm{a}}$, except along the special direction defined by a simultaneous and equal change in all variables, i.e., $\theta_{m}=\phi / N$ for all array variables. Such collective dynamics is associated with the black-sheep variable and has a barrier height of only $2 E_{\mathrm{J}}^{\mathrm{b}}$. In the quantum regime, fluctuations will occur primarily along this direction and motivate the use of $\phi$ as an essential collective variable.

For the symmetric-fluxonium model, this collective motion of all array variables forms a normal mode for harmonic oscillations around the global minimum that, for zero magnetic flux, is located at $\phi=0$. Anticipating the role of this mode, we refer to it as the superinductance mode. Further analysis shows that the remaining $N-1$ normal modes are degenerate, and, so as to remain orthogonal to the superinductance mode, their amplitudes sum to zero mode by mode. We therefore call them difference modes and introduce $\xi_{\mu}(\mu=1, \ldots, N-1)$ as their amplitude variables (see Fig. 2). The transformation to the new set of variables $\left\{\phi, \xi_{1}, \ldots, \xi_{N-1}\right\}$ is facilitated by

$$
\theta_{m}=\phi / N+\sum_{\mu} W_{\mu m} \xi_{\mu}
$$

and, inversely, $\phi=\sum_{m} \theta_{m}$ and $\xi_{\mu}=\sum_{m} W_{\mu m} \theta_{m}$. Unless otherwise specified, sums over Greek indices run over the difference modes $\mu=1, \ldots, N-1$. The $(N-1) \times N$ matrix $W$ is semiorthogonal, and its components sum up to zero in each row, i.e., $\sum_{m} W_{\mu m} W_{\nu m}=\delta_{\mu \nu}$ and $\sum_{m} W_{\mu m}=0$. Our choice

$$
W_{\mu m}=\sqrt{2 / N} \cos \left[\pi \mu\left(m-\frac{1}{2}\right) / N\right]
$$

differs from the choice in Ref. [26]. It proves particularly convenient for the subsequent discussion of corrections

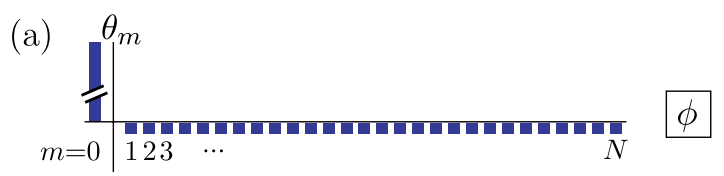

(b)

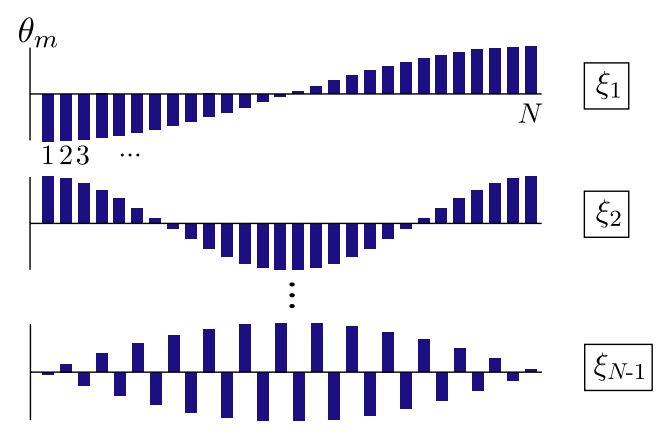

FIG. 2. Normal modes for the $S_{N}$ symmetric-fluxonium model. The plots show the array-variable amplitudes $\theta_{m}$ for each normal mode. (a) Superinductance mode $[\phi]$, for which all arrayjunction amplitudes $\theta_{m}$ are identical. (b) Difference modes $\left[\xi_{\mu}\right]$, for all of which the amplitude sum exactly vanishes. from ground capacitances [see the paragraphs containing Eqs. (9) and (12)] that break $S_{N}$ symmetry. After this variable transformation, circuit quantization yields the symmetric-fluxonium Hamiltonian

$$
\begin{aligned}
H_{\mathrm{SFM}}= & -4 E_{\mathrm{C}}^{\mathrm{si}} \partial_{\phi}^{2}-4 E_{\mathrm{C}}^{\mathrm{a}} \sum_{\mu} \partial_{\xi_{\mu}}^{2}-E_{\mathrm{J}}^{\mathrm{b}} \cos \left(\phi+\varphi_{\mathrm{ext}}\right) \\
& -E_{\mathrm{J}}^{\mathrm{a}} \sum_{m} \cos \left[\phi / N+\sum_{\mu} W_{\mu m} \xi_{\mu}\right]
\end{aligned}
$$

where $E_{\mathrm{C}}^{\mathrm{si}}=E_{\mathrm{C}}^{\mathrm{b}} /\left[1+E_{\mathrm{C}}^{\mathrm{b}} /\left(E_{\mathrm{C}}^{\mathrm{a}} N\right)\right]$ is equal to the blacksheep charging energy up to a small $1 / N$ correction.

The structure of $H_{\mathrm{SFM}}$ illustrates the utility of the collective-mode description: Coupling between different modes is limited to potential-energy terms, and the "effective masses" are identical for all difference-mode amplitudes. Further, at the relevant potential minima, all difference-mode amplitudes vanish, $\xi_{\mu}=0$, and the arguments of the array cosines [last line of Eq. (5)] are of order $1 / N$. Hence, a Taylor expansion for small arguments can be expected to capture the essential low-energy physics. Keeping terms up to second order in this expansion, one obtains

$$
H_{0}=-4 E_{\mathrm{C}}^{\mathrm{si}} \partial_{\phi}^{2}-E_{\mathrm{J}}^{\mathrm{b}} \cos \left(\phi+\varphi_{\mathrm{ext}}\right)+\frac{E_{L}}{2} \phi^{2}+\sum_{\mu} \Omega a_{\mu}^{\dagger} a_{\mu},
$$

where $a_{\mu}^{\dagger}=\left(\xi_{\mu} / \Delta_{\xi}-\Delta_{\xi} \partial_{\xi_{\mu}}\right) / \sqrt{2}$ is the ladder operator that creates an excitation in the $\mu$ th difference mode, $\Delta_{\xi}=$ $\left(8 E_{\mathrm{C}}^{\mathrm{a}} / E_{\mathrm{J}}^{\mathrm{a}}\right)^{1 / 4}$ is the oscillator length, and $\Omega=\sqrt{8 E_{\mathrm{C}}^{\mathrm{a}} E_{\mathrm{J}}^{\mathrm{a}}}$ is the array-junction plasma frequency. Eigenstates of $H_{0}$ take the form of direct-product states $|l\rangle_{\mathrm{s}} \otimes|\vec{s}\rangle_{\mathrm{d}}$. In this expression, $l=0,1, \ldots$ enumerates the superinductance eigenstates (variable $\phi$ ), and the components of the $(N-1)$-dimensional vector $\vec{s}$ denote the occupation numbers of the difference modes, i.e.,

$$
|\vec{s}\rangle_{\mathrm{d}}=\prod_{\mu=1}^{N-1} \frac{\left(a_{\mu}^{\dagger}\right)^{s_{\mu}}}{\sqrt{s_{\mu} !}}|\overrightarrow{0}\rangle_{\mathrm{d}}
$$

The first three terms in the expression for $H_{0}$ reproduce the superinductance model that was successfully used in Refs. [8,39]. It describes the superinductance mode as the coupled system of the black-sheep junction with capacitive energy $E_{\mathrm{C}}^{\mathrm{si}}$ and a large superinductance [40] $L_{s}=$ $N\left(\Phi_{0} / 2 \pi\right)^{2} / E_{\mathrm{J}}^{\mathrm{a}}$ with correspondingly small inductive energy $E_{L}=E_{\mathrm{J}}^{\mathrm{a}} / N$. As the second crucial insight from $H_{0}$, we note that, within the harmonic approximation, the symmetry of the circuit has been extended to include arbitrary unitary transformations of the $N-1$ degenerate difference modes. As the superinductance mode is a scalar under the action of the group $\mathrm{U}(N-1)$, it completely decouples from the difference modes in the harmonic limit. This decoupling explains, in part, the success of the superinductance model in matching experimental spectra in spite of the presence of the large number of additional 
degrees of freedom. The concept of symmetry-induced decoupling carries over to more complicated circuits that include linear arrays of Josephson junctions.

\section{WEAK $S_{N}$ SYMMETRY BREAKING}

The symmetric-fluxonium model $H_{\mathrm{SFM}}$ and its approximation $H_{0}$ both obey $\mathrm{S}_{N}$ symmetry. The symmetries of $H_{0}$ are enlarged by the harmonic approximation and include an additional $\mathrm{U}(N-1)$ symmetry in the difference-mode subspace: Any transformation $a_{\mu} \rightarrow \sum_{\nu} U_{\mu \nu} a_{\nu}$ with unitary $U$ leaves $H_{0}$ invariant. To go beyond the superinductance model and predict corrections arising from the weak interaction between the superinductance mode and the difference modes, we next consider mechanisms that lead to symmetry breaking.

As is summarized in Table I, we focus on the following three mechanisms that are likely the dominant ones in the present experimental samples: anharmonicities of the potential energy neglected in the above expansion $\left(\delta H_{U}\right)$, disorder in the Josephson energies of individual array junctions $\left(\delta H_{\mathrm{J}}\right)$, and additional stray capacitances of each superconducting island to ground $\left(\delta H_{\mathrm{C}}\right)$. We first derive the Hamiltonian expressions for each of these corrections and subsequently discuss their effects on the energy spectrum and eigenstates.

We start with $\delta H_{U}$, the corrections from anharmonicities exhibited by the periodic potential but neglected in the harmonic approximation employed in $H_{0}$. Considering higher-order terms in the Taylor expansion of $H_{\mathrm{SFM}}-$ $H_{0}$, we find that the leading anharmonic corrections are given by

$$
\delta H_{U}=-\frac{E_{\mathrm{J}}^{\mathrm{a}}}{4 !} \sum_{m}\left(\phi / N+\sum_{\mu} W_{\mu m} \xi_{\mu}\right)^{4} .
$$

It is easy to verify that $\delta H_{U}$ breaks the $\mathrm{U}(N-1)$ symmetry but preserves the permutation symmetry under $\mathrm{S}_{N}$.
To derive an expression for $\delta H_{\mathrm{J}}$, we capture disorder in the Josephson energies of the array by defining $E_{\mathrm{J} m}=$ $E_{\mathrm{J}}^{\mathrm{a}}+\delta E_{\mathrm{J} m}$. Such disorder is expected to be caused by slight variations in junction size and thickness and may also be affected by junction aging. In the absence of experimental statistics for fluxonium-junction parameters, we choose random $\delta E_{\mathrm{J} m}$ from a Gaussian distribution of width $\delta E_{\mathrm{J}}=150 \mathrm{MHz}$ and, without loss of generality, impose $\sum_{m} \delta E_{\mathrm{J} m}=0$. The disorder modifies the potential energy of the Hamiltonian, and through Taylor expansion we obtain

$$
\delta H_{\mathrm{J}}=\frac{1}{2} \sum_{m} \delta E_{\mathrm{J} m}\left(\phi / N+\sum_{\mu} W_{\mu m} \xi_{\mu}\right)^{2}
$$

Disorder in individual array-junction parameters generally leads to weak breaking of both $\mathrm{U}(N-1)$ and $\mathrm{S}_{N}$ symmetry.

To capture corrections from stray capacitances of the superconducting islands to ground, we include the terms due to ground capacitances shown in Eq. (1). Ground capacitances contribute kinetic-energy terms that are easily expressed as $T_{j}=\frac{1}{2}\left(\Phi_{0} / 2 \pi\right)^{2} C_{\mathrm{g} j} \dot{\varphi}_{j}^{2}$ when using node variables $\varphi_{j}$ for each superconducting island. Assuming overall charge neutrality of the circuit, we can recast these additional contributions in terms of the junction variables $\dot{\theta}_{m}$. Accounting for the ground capacitances of the two large superconducting islands surrounding the black sheep and the ground capacitances of the remaining small islands by $C_{\mathrm{g}}^{\mathrm{b}}, C_{\mathrm{g}}^{\mathrm{a}} \ll C_{\mathrm{J}}^{\mathrm{a}}, C_{\mathrm{J}}^{\mathrm{b}}$ (Fig. 1), the perturbation can be expressed as

$$
\delta H_{\mathrm{C}} \simeq 4 \sum_{\mu, \nu=0}^{N-1}\left(M^{-1} G M^{-1}\right)_{\mu \nu} \partial_{\xi_{\mu}} \partial_{\xi_{\nu}},
$$

where $\partial_{\xi_{0}}=\partial_{\phi}$ and $\partial_{\xi_{\mu}}=\left(a_{\mu}-a_{\mu}^{\dagger}\right) /\left(\sqrt{2} \Delta_{\xi}\right)$ for $\mu \geq 1$. The detailed derivation of Eq. (9) and analytical expressions for

TABLE I. Summary of the principal effects of the perturbations $\delta H_{U}, \delta H_{\mathrm{C}}$, and $\delta H_{\mathrm{J}}$ organized by the type of coupling. The three

\begin{tabular}{|c|c|c|c|}
\hline $\begin{array}{l}\text { Perturbation } \\
\text { origin } \rightarrow \\
\text { and type } \downarrow\end{array}$ & Anharmonicity $\left(\delta H_{U}\right)$ & Capacitance to ground $\left(\delta H_{\mathrm{C}}\right)$ & Josephson-energy disorder $\left(\delta H_{\mathrm{J}}\right)$ \\
\hline s & Renormalize $E_{L}$ [Eq. (10)]. & Renormalize $E_{\mathrm{C}}^{\text {si }}[$ Eq. (10)]. & \\
\hline $\mathrm{d}$ & $\begin{array}{l}\text { Reduces symmetry from } \mathrm{U}(N-1) \\
\text { to } \mathrm{S}_{N} \text { and splits subspaces into } \\
\text { irreducible components } \\
\mathcal{V}_{\mathrm{T}} \rightarrow \mathcal{V}_{\mathrm{T}(\lambda)}[\text { Fig. } 4(\mathrm{~b})]\end{array}$ & $\begin{array}{l}\text { Reduces symmetry from } \mathrm{S}_{N} \text { to } \\
P T \text { and generates the largest } \\
\text { energy shift for difference modes } \\
\text { with small } \mu \text { [Eq. (12)]. }\end{array}$ & $\begin{array}{l}\text { Removes all symmetries and } \\
\text { broadens the energy distribution } \\
\text { of the difference modes [Fig. } 5(\mathrm{~b}) \text { ]. }\end{array}$ \\
\hline sd & $\begin{array}{l}\text { Symmetry-enforced decoupling } \\
\text { of subspaces that are inequivalent } \\
\text { with respect to } S_{N} \text { symmetry } \\
\text { [Fig. } 5 \text { (a)]. }\end{array}$ & $\begin{array}{l}\text { Creates coupling between the } \\
\text { superinductor and the even } \\
\text { difference modes [Eq. (13)]. }\end{array}$ & $\begin{array}{l}\text { Creates coupling between the } \\
\text { superinductor and all the difference } \\
\text { modes [Eq. (14)]. }\end{array}$ \\
\hline
\end{tabular}
types of coupling are "s," coupling among superinductance states; "d," coupling among difference-mode states; and "sd," coupling between the two subsystems. 
the entries of the matrices $M$ and $G$ are provided in Appendix D.

After removing irrelevant global-energy shifts, the effects of the perturbations $\delta H_{U}, \delta H_{\mathrm{C}}$, and $\delta H_{\mathrm{J}}$ can be organized into three categories according to their action on the superinductance and difference modes. Perturbations may introduce coupling among superinductance states $\left(\delta H_{\bullet}^{\mathrm{s}}\right)$ and coupling among difference-mode states $\left(\delta H_{\bullet}^{\mathrm{d}}\right)$, as well as coupling between the two subsystems $\left(\delta H_{\bullet}^{\text {sd }}\right)$.

We first discuss corrections in the $\delta H_{\text {. }}^{\text {s category, which }}$ only affect the superinductance mode. The simplest contributions of this type are terms with a structure identical to those already present in $H_{0}$ that merely renormalize the superinductance model parameters. Both $\delta H_{U}$ and $\delta H_{\mathrm{C}}$ contain corrections of this type and yield renormalized parameters

$$
E_{\mathrm{C}}^{\mathrm{si}} \rightarrow \frac{1}{1 / E_{\mathrm{C}}^{\mathrm{b}}+1 / N E_{\mathrm{C}}^{\mathrm{a}}+G_{00}}, \quad E_{L} \rightarrow \frac{E_{\mathrm{J}}^{\mathrm{a}}}{N}\left[1-\Delta_{\xi}^{2} \frac{N-1}{4 N}\right] .
$$

We emphasize that the inclusion of ground capacitances of the large islands alone leaves the $S_{N}$ symmetry unharmed and is completely accounted for in our model by the above renormalization, even in the limit that $C_{\mathrm{g}}^{\mathrm{b}}$ is large. The only contribution of type $\delta H^{\mathrm{s}}$. that goes beyond renormalization is the term $-E_{\mathrm{J}}^{\mathrm{a}} \phi^{4} /\left(4 ! N^{3}\right)$ generated by $\delta H_{U}$. For realistic parameters, we find that this perturbation generates level shifts well below $100 \mathrm{MHz}$ (see Sec. IV).

We next turn to corrections in the $\delta H_{\text {. }}^{\mathrm{d}}$ category to discern how anharmonicity $\delta H_{U}$, ground capacitance $\delta H_{\mathrm{C}}$, and array-junction disorder $\delta H_{\mathrm{J}}$ affect the spectrum of difference-mode excitations. Perturbations from anharmonicity $\delta H_{U}$ break the $\mathrm{U}(N-1)$ symmetry but leave the $\mathrm{S}_{N}$ symmetry subgroup intact. As a result, degeneracies are lifted only partially and degenerate perturbation theory must be used. Each remaining degenerate subspace is associated with an irreducible representation of the symmetric group. Our construction of the relevant irreducible subspaces works as follows. We start by decomposing the difference-mode Hilbert space into orthogonal subspaces $\mathcal{V}_{\mathrm{T}}$ with fixed excitation number $\mathrm{T}=\sum_{\mu} a_{\mu}^{\dagger} a_{\mu}$, i.e., $\mathcal{H}_{\mathrm{d}}=\mathcal{V}_{0} \oplus \mathcal{V}_{1} \oplus \cdots$. In general, each $\mathcal{V}_{\mathrm{T}}$ may still be reducible under $\mathrm{S}_{N}$ and should be further decomposed.

In this decomposition, the integer partitions of $N$ serve as labels for the irreducible representations of the symmetric group $\mathrm{S}_{N}$. Here, a partition $(\lambda)=\left(\lambda_{1}, \lambda_{2}, \ldots, \lambda_{F}\right)$ is a sequence of nonincreasing positive integers

$$
\lambda_{1} \geq \lambda_{2} \geq \cdots \geq \lambda_{F}>0
$$

that sum to $N$. Each partition is conveniently represented by a Young diagram: a collection of $N$ boxes arranged in $F$ left-justified rows with the $i$ th row having the length $\lambda_{i}$. For $N=6$, the partition $(4,2)$ is represented by the Young diagram
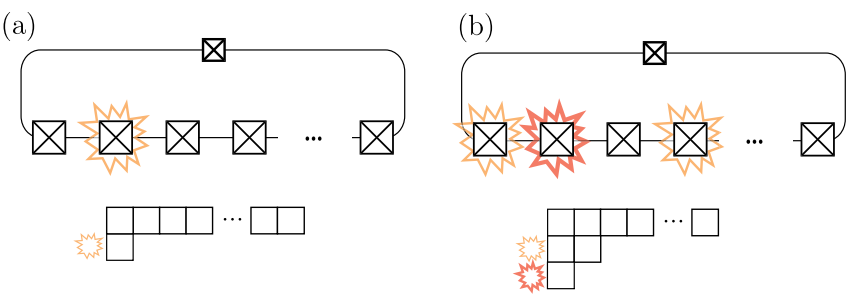

FIG. 3. Physical interpretation of Young diagrams. (a) Each subspace labeled by a Young diagram of shape $(N-1,1)$ is spanned by $N$ states in which one array junction is excited relative to the other $N-1$ array junctions. These states are linearly dependent and can be decomposed into differencemode excitations. For example, the irreducible $(N-1,1)$ subspace $V_{1}$ has a basis that is comprised of the $N-1$ differencemode excitations $a_{\mu}^{\dagger}|\overrightarrow{0}\rangle_{\mathrm{d}}$ that are collective excitations distributed across multiple junctions (see Fig. 2). (b) Subspaces with higher junction excitations are associated with Young diagrams with additional rows.

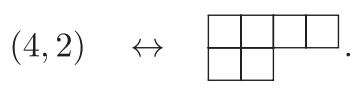

Since the inductive decomposition of $\mathrm{S}_{N}$ [41] is not very practical for $N \gg 1$, we decompose the subspaces $\mathcal{V}_{\mathrm{T}}$ by using a restricted set of semistandard Young tableaux [42]. (All technical details of this procedure are provided in Appendix A.) For the low-energy part of the spectrum probed by experiments, we find that the excitation number $\mathrm{T}$ and partition $(\lambda)$ are sufficient to specify the relevant irreducible subspaces. A simplified physical interpretation of the Young diagrams is offered in Fig. 3.

The subspace without any difference-mode excitations, $\mathcal{V}_{\mathrm{T}=0}$, is spanned by only the ground state $|\overrightarrow{0}\rangle_{\mathrm{d}}$. It immediately forms an irreducible representation. The state is effectively bosonic and is indexed by the partition $(\lambda)=$ $(N)$. The subspace of difference-mode states with a single excitation, $\mathcal{V}_{\mathrm{T}=1}=\operatorname{span}\left\{a_{\mu}^{\dagger}|\overrightarrow{0}\rangle_{\mathrm{d}} \mid \mu=1,2, \ldots, N-1\right\}$, already forms an irreducible $N-1$-dimensional subspace corresponding to the representation with partition $(\lambda)=$ $(N-1,1)$ (Fig. 4). For $\mathrm{T}=2$, the decomposition is more interesting and results in three irreducible subspaces indexed by $(N),(N-1,1)$, and $(N-2,2)$. The subspace labeled by $(N)$, for example, is comprised of a single $\mathrm{S}_{N}$-invariant state given by

$$
|\mathrm{T}(\lambda)\rangle=|2(N)\rangle=\frac{1}{\sqrt{2(N-1)}} \sum_{\mu}\left(a_{\mu}^{\dagger}\right)^{2}|\overrightarrow{0}\rangle_{\mathrm{d}},
$$

which is independent of the specific choice of $W$ in Eq. (3). By employing perturbation theory for $\delta H_{U}^{\mathrm{d}}$ in each irreducible subspace $\mathcal{V}_{\mathrm{T}(\lambda)}$, we obtain the first-order energy corrections $\delta E_{U}^{\mathrm{d}}[\mathrm{T},(\lambda)]$ in Table II. The resulting level shifts are shown schematically in Figs. 4(a) and 4(b).

We next consider perturbations that break the $\mathrm{S}_{N}$ symmetry and thus lift the degeneracy of difference modes. Both corrections from ground capacitances, $\delta H_{\mathrm{C}}^{\mathrm{d}}$, and from junction disorder, $\delta H_{\mathrm{J}}^{\mathrm{d}}$, fall in this category. If the ground 


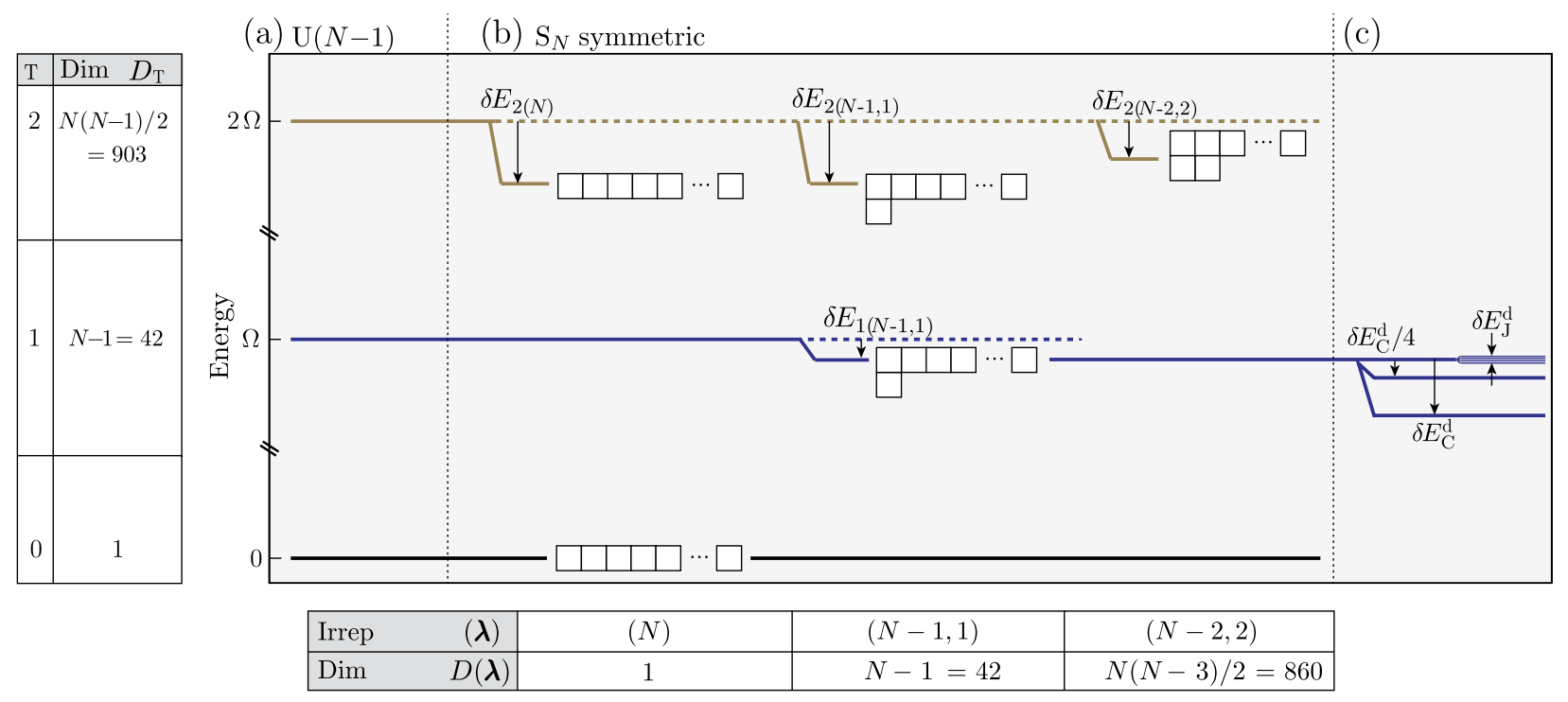

FIG. 4. Difference-mode spectrum for total excitation numbers $\mathrm{T}=0,1,2$. (a) Within the harmonic approximation, $\mathrm{S}_{N}$ and $\mathrm{U}(N-1)$ symmetry hold and produce degenerate subspaces of dimension (dim) $D_{\mathrm{T}}$. (b) Anharmonicity $\delta H_{U}$ breaks $\mathrm{U}(N-1)$ but leaves $\mathrm{S}_{N}$ symmetry intact. The irreducible representations (irreps) of $\mathrm{S}_{N}$, labeled by partitions $(\lambda)$ or Young diagrams, give rise to $D(\lambda)$-dimensional degenerate subspaces. (c) Corrections from disorder in array-junction $E_{\mathrm{J}}$ 's and ground capacitances, $\delta H_{\mathrm{C}}+\delta H_{\mathrm{J}}$, break $\mathrm{S}_{N}$ symmetry. The degeneracy lifting is shown for the $\mathrm{T}=1$ subspace.

capacitance of the big islands is sufficiently large compared to that of the small islands, $E_{\mathrm{g}}^{\mathrm{b}} / E_{\mathrm{g}}^{\mathrm{a}} \ll 1 / N^{2}$, then $\delta H_{\mathrm{C}}^{\mathrm{d}}$ in Eq. (9) is approximately diagonal. This decoupling is the primary motivation for our choice of difference modes [Eq. (3)]. To leading order, the resulting energy shifts for states in the in the one-excitation subspace $\mathcal{V}_{1}$ are given by

$$
\delta E_{\mathrm{C}, \mu}^{\mathrm{d}} \approx-\delta E_{\mathrm{C}}^{\mathrm{d}} / \mu^{2} \quad(\mu \ll N),
$$

where $\delta E_{\mathrm{C}}^{\mathrm{d}}=4 N^{2} E_{\mathrm{C}}^{\mathrm{a} 2} /\left(\pi^{2} \Delta_{\xi}^{2} E_{\mathrm{g}}^{\mathrm{a}}\right)$. Similar effects from ground capacitances have been predicted and observed in Ref. [16]. For higher values of $\mu$, shifts induced by Josephson-energy disorder $\delta H_{\mathrm{J}}^{\mathrm{d}}$ become dominant in our model. For Gaussian-distributed junction parameters $\delta E_{\mathrm{J} m}$, the first-order energy shifts $\delta E_{\mathrm{J}, \mu}^{\mathrm{d}}$ also follow a Gaussian distribution with width $\delta E_{\mathrm{J}}^{\mathrm{d}}=\delta E_{\mathrm{J}} \Delta_{\xi}^{2} / 2$ [Figs. 4(c) and 5(b)].

TABLE II. Energy corrections for difference-mode states due to anharmonicities $\left(\delta H_{U}\right)$. T is the total number of excitations in the difference modes, and $(\lambda)$ is the partition labeling the irreducible subspace.

\begin{tabular}{lcc}
\hline \hline $\mathrm{T}$ & $(\lambda)$ & $-\delta E_{U}^{\mathrm{d}}[\mathrm{T},(\lambda)] / E_{\mathrm{C}}^{\mathrm{a}}$ \\
\hline 0 & $(N)$ & 0 \\
1 & $(N-1,1)$ & $1-1 / N$ \\
2 & $(N)$ & $3-3 / N$ \\
2 & $(N-1,1)$ & $3-4 / N$ \\
2 & $(N-2,2)$ & $2-2 / N$ \\
\hline \hline
\end{tabular}

Interesting corrections in the third and final category, $\delta H_{\bullet}^{\text {sd }}$, arise from coupling between superinductance and difference modes. Anharmonicity captured by $\delta H_{U}^{\text {sd }}$ preserves $\mathrm{S}_{N}$ symmetry and hence, by Schur's lemma, cannot couple states belonging to different irreducible representations. More specifically, states of the form $\left|\ell^{\prime}\right\rangle_{\mathrm{s}} \otimes a_{\mu}^{\dagger}|\overrightarrow{0}\rangle_{\mathrm{d}}$ cannot couple to states of the form $|\ell\rangle_{\mathrm{s}} \otimes|\overrightarrow{0}\rangle_{\mathrm{d}}$ under $\mathrm{S}_{N}$ symmetry, even when such states are degenerate [Fig. 5(a)]. This symmetry-enforced lack of coupling between the superinductance mode and the lowest differencemode excitations constitutes a central result of our work. It is a crucial ingredient in preserving the respective identity of these collective modes and explains the quantitative accuracy of the superinductance model at low energies. The only difference-mode excitations that may couple to states of the form $|\ell\rangle_{\mathrm{s}} \otimes|\overrightarrow{0}\rangle_{\mathrm{d}}$ are those that are bosonic, i.e., that are indexed by the partition $(N)$. The candidate states with lowest energies are $\left|\ell^{\prime}\right\rangle_{\mathrm{s}} \otimes|2(N)\rangle$ but are already well beyond the frequency range probed by spectroscopy in previous fluxonium experiments.

Ground capacitances, as described by $\delta H_{\mathrm{C}}^{\mathrm{sd}}$, break $\mathrm{S}_{N}$ symmetry but preserve $P T$ symmetry: $\varphi_{j} \rightarrow-\varphi_{N-j}$. $P T$ symmetry is a combination of "circuit parity" $P$ that mirrors the circuit variables and time reversal $T . P T$ is a symmetry of $H_{0}$ and $H_{\mathrm{SFM}}$, even for nonzero flux $\varphi_{\mathrm{ext}}$. The superinductance mode and difference modes with even index $\mu$ are even under $P T$; difference modes with odd index $\mu$ are odd under $P T$. As a result, $\delta H_{\mathrm{C}}^{\text {sd }}$ can only couple the superinductance mode to every other difference mode. We find that the coupling is largest for small values of $\mu$ and takes the form 

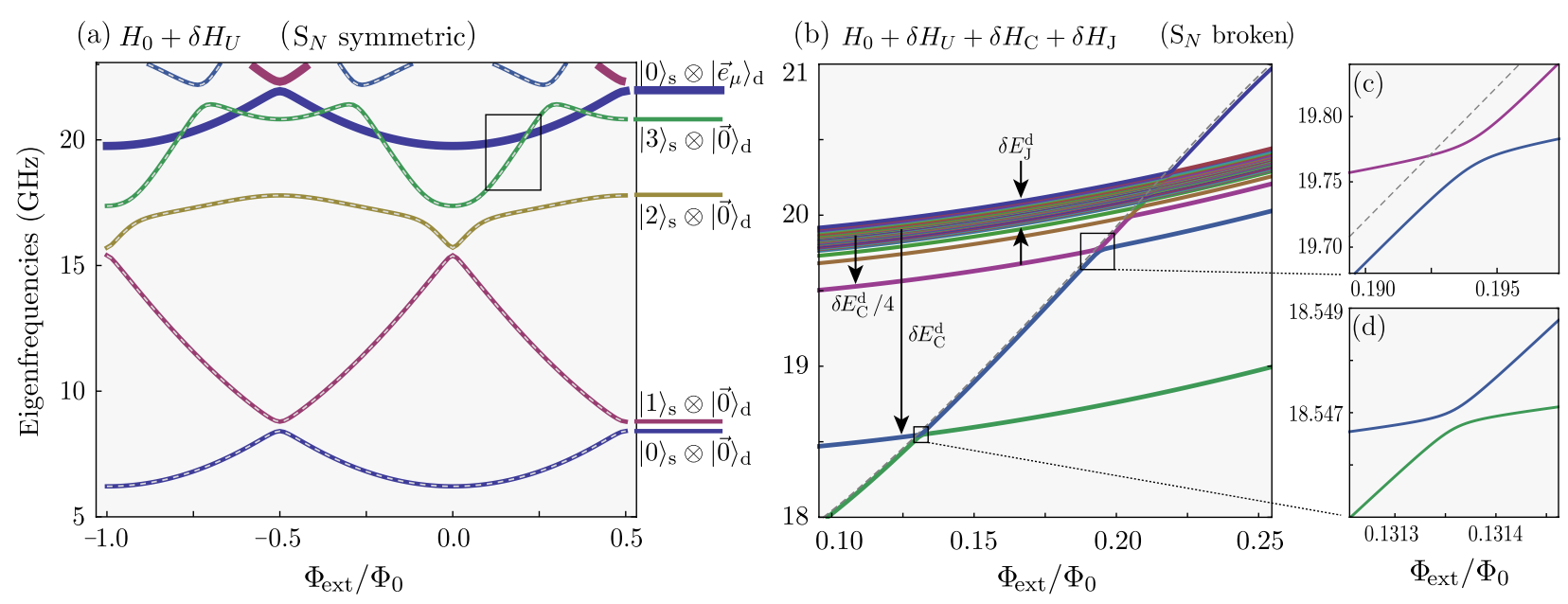

FIG. 5. Spectrum from numerical diagonalization, including the effects of (a) anharmonicity and of (b) anharmonicity, ground capacitances, and junction disorder. The dashed curves show the pure superinductance spectra for renormalized $E_{\mathrm{C}}^{\mathrm{si}}$ and $E_{L}$ [Eq. (10)]. The thick curves in (a) indicate $N-1$-fold degenerate levels that remain decoupled from $\mathrm{T}=0$ states under $\delta H_{U}$. (b) Corrections from ground capacitance $\delta H_{\mathrm{C}}$ and junction disorder $\delta H_{\mathrm{J}}$ break $\mathrm{S}_{N}$ symmetry. In the $\mathrm{T}=1$ manifold, ground capacitances split off several levels; smaller shifts are due to junction disorder $\delta E_{\mathrm{J} \mu}^{\mathrm{d}}$, here chosen from a Gaussian distribution. (c) and (d) show magnifications of regions marked in (b). The avoided crossing in (c) is primarily generated by ground capacitances. The even smaller splitting in (d) is purely generated by array-junction disorder. The chosen parameters are consistent with the experimental device (Appendix F).

$$
\delta H_{\mathrm{C}}^{\mathrm{sd}} \approx-\partial_{\phi} \sum_{\mu=2,4,6 \ldots} \delta E_{\mathrm{C}}^{\mathrm{sd}}\left(a_{\mu}-a_{\mu}^{\dagger}\right) / \mu^{2},
$$

where $\delta E_{\mathrm{C}}^{\mathrm{sd}}=8 E_{\mathrm{C}}^{\mathrm{a}} E_{\mathrm{C}}^{\mathrm{si}} N^{3 / 2} /\left(\pi^{2} \Delta_{\xi} E_{\mathrm{g}}^{\mathrm{a}}\right)$. Finally, all symmetries are broken for array-junction disorder, and the resulting perturbation is given by

$$
\delta H_{\mathrm{J}}^{\mathrm{sd}} \approx \phi \sum_{\mu} \delta E_{\mathrm{J} \mu}^{\mathrm{sd}}\left(a_{\mu}+a_{\mu}^{\dagger}\right),
$$

where $\delta E_{\mathrm{J}, \mu}^{\mathrm{sd}}$ follows a Gaussian distribution of width $\delta E_{\mathrm{J}} \Delta_{\xi} /(\sqrt{2} N)$. As shown in Figs. 5(c) and 5(d), the coupling between superinductance and the $\mu=1$ difference mode, induced by $\delta H_{\mathrm{J}}^{\text {sd }}$ only, is considerably smaller than the coupling to the $\mu=2$ difference mode that is dominated by $\delta H_{\mathrm{C}}^{\text {sd }}$.

\section{DISCUSSION AND SUMMARY}

The low-energy spectrum of the full fluxonium circuit includes, in addition to the energy levels predicted by the superinductance model, a large number of nearly degenerate excitations. We have identified the nature of these collective excitations with the difference modes at energies near the array-junction plasma frequency. Degeneracies are expected to be lifted, first by $\mathrm{U}(N-1)$ symmetry breaking due to anharmonicity and further by $\mathrm{S}_{N}$ symmetry breaking due to array-junction disorder and ground capacitances. The important consequences of these corrections include separation of previously degenerate levels into closely spaced multiplets.

Josephson-junction arrays provide an interesting example of a quantum system with many identical but distinguishable degrees of freedom, resulting in representations of the symmetric group that are not readily observed in nature with indistinguishable particles. Invariance under permutations of the junction variables is a generic symmetry that is expected to be important for any large superconducting circuit containing one or several Josephson-junction arrays. The decomposition of the symmetric group $S_{N}$ into irreducible representations relevant at low energies thus becomes an important tool in circuit analysis. For the example of the fluxonium device, we have shown that such symmetry strongly restricts the possible coupling between the superinductance mode, as observed in the experiment [8,9], and the additional difference modes. Our results explain the remarkable accuracy of the effective superinductance model as long as the renormalizations of $E_{L}$ and $E_{C}^{\text {si }}$ are taken into account and are consistent with previous fits of experimental data where $E_{L}$ and $E_{\mathrm{C}}^{\mathrm{si}}$ were used as fit parameters, producing excellent agreement $[8,9]$.

The power of symmetry-based approaches in the analysis of future circuits is easily illustrated for the example of the fluxonium device. Specifically, the number of differencemode states with excitations up to some threshold $\mathrm{T}$ grows rapidly as $\frac{(\mathrm{T}+N-1) !}{\mathrm{T}(N-1) !}$. The number of states with proper bosonic symmetry, however, is dramatically smaller: For $N=43$ and $\mathrm{T}=5$, there are $10^{6}$ difference-mode states, but merely six of them possess bosonic symmetry. Harnessing exact and approximate symmetries of Hamiltonians for larger circuits will likely be a crucial ingredient in future research that explores quantum coherence in superconducting circuit networks of increasing complexity. 


\section{ACKNOWLEDGMENTS}

We thank Leonid Glazman, Michel Devoret, and Vladimir Manucharyan for stimulating discussions. Our research was supported by the NSF under Grants No. PHY-1055993 (J. K., D. G. F.) and No. DMR0805277 (D. G. F.) and by the David and Lucile Packard Foundation (A. A. H).

\section{APPENDIX A: IRREDUCIBLE REPRESENTATIONS FOR DIFFERENCE MODES}

In this Appendix, we discuss the decomposition of the difference-mode Hilbert spaces $\mathcal{V}_{\mathrm{T}}$ into subspaces that transform irreducibly under $S_{N}$ symmetry. Since some of the mathematical tools employed may not belong to the physicist's ordinary repertoire, we provide definitions along with concrete examples where appropriate. In terminology and notation, our discussion closely follows the excellent book by Sagan [42].

The subspace $\mathcal{V}_{\mathrm{T}}$ comprises all difference-mode states with total excitation number T. It is spanned by the orthogonal states

$$
a_{\mu_{1}}^{\dagger} \cdots a_{\mu_{\mathrm{T}}}^{\dagger}|\overrightarrow{0}\rangle,
$$

where we assume weakly ordered mode indices

$$
\mu_{1} \leq \cdots \leq \mu_{\mathrm{T}} \in\{1,2, \ldots, N-1\}
$$

to avoid double counting. The $\mathrm{S}_{N}$ symmetry displayed in the ideal fluxonium circuit pertains to permutations $\sigma \in \mathrm{S}_{N}$ of the array-junction variables $\theta_{1}, \theta_{2}, \ldots, \theta_{N}$. Such permutations also induce linear transformations in the operator space $\mathbb{C}\left\{a_{1}^{\dagger}, a_{2}^{\dagger}, \ldots, a_{N-1}^{\dagger}\right\}$ spanned by the difference-mode creation operators. To understand how $a_{\mu}^{\dagger}$ transforms under permutations, we recall the definition of the creation operators in terms of junction variables:

$$
a_{\mu}^{\dagger}=\sum_{m} W_{\mu m}\left(\theta_{m} / \Delta_{\xi}-\Delta_{\xi} \partial_{\theta_{m}}\right) / \sqrt{2}
$$

Using the identity $\sum_{\mu} W_{\mu m} W_{\mu n}=\delta_{m n}-\frac{1}{N}$, one finds that the difference-mode creation operators transform according to

$$
\sigma\left(a_{\mu}^{\dagger}\right)=\sum_{\nu} S(\sigma)_{\mu \nu} a_{\nu}^{\dagger}
$$

We remind the reader that, by our convention, sums with Latin (Greek) summation indices always range from 1 to $N$ $(1$ to $N-1)$. The $(N-1) \times(N-1)$ transformation matrices are given by

$$
S(\sigma)_{\mu \nu}=\sum_{m, n} W_{\mu m} W_{\nu n} D(\sigma)_{m n} .
$$

Here, $D(\sigma)_{m n}=\delta_{m, \sigma(n)}$ denotes the $N \times N$ permutation matrix for the group element $\sigma \in \mathrm{S}_{N}$. [The matrices $D(\sigma)$ form the defining representation of $S_{N}$.]
By the relation $\mathcal{V}_{1}=\mathbb{C}\left\{a_{1}^{\dagger}, a_{2}^{\dagger}, \ldots, a_{N-1}^{\dagger}\right\}|\overrightarrow{0}\rangle$, the transformation matrices $S(\sigma)$ in Eq. (A3) define an orthogonal $\mathrm{S}_{N}$ representation of dimension $N-1$ in the oneexcitation subspace. Similarly, for higher excitation numbers $\mathrm{T}>1$, the group action for products of creation operators,

$$
\sigma\left(a_{\mu_{1}}^{\dagger} \cdots a_{\mu_{\mathrm{T}}}^{\dagger}\right)=\sum_{\nu_{1}, \ldots, \nu_{\mathrm{T}}} S(\sigma)_{\mu_{1} \nu_{1}} \cdots S(\sigma)_{\mu_{\mathrm{T}} \nu_{\mathrm{T}}} a_{\nu_{1}}^{\dagger} \cdots a_{\nu_{\mathrm{T}}}^{\dagger},
$$

determines the representation of $S_{N}$ in the subspace $\mathcal{V}_{\mathrm{T}}$. Given these representations, our central task is to decompose each $\mathcal{V}_{\mathrm{T}}$ into its irreducible subspaces. As an aside, we note that, in the special case of $\mathcal{V}_{1}$, simple arguments based on group characters can be used to show that $\mathcal{V}_{1}$ is already irreducible and coincides with the irreducible representation indexed by the partition $(N-1,1)$, for which group characters are known to be $\operatorname{tr} D(\sigma)-1$ for arbitrary $N$ (Ref. [42], Sec. 2.12).

The common approach for the decomposition of such product representations is inductive and requires successive decompositions for $\mathrm{S}_{1}, \mathrm{~S}_{2}, \ldots, \mathrm{S}_{N}$; see, e.g., Ref. [41]. For large $N$, however, that strategy is not very practical. Following the treatment by Sagan [42], we thus employ an alternate approach using a restricted class of semistandard tableaux. (We will explain the meaning of these words in this Appendix, Step 2.)

As our first step in constructing the decomposition of each $\mathcal{V}_{\mathrm{T}}$, we define the pseudocreators $b_{n}^{\dagger}$ for $n=$ $1,2, \ldots, N$ by

$$
b_{n}^{\dagger}=\sum_{\mu} W_{\mu n} a_{\mu}^{\dagger}=\left[\left(\theta_{n}-\phi / N\right) / \Delta_{\xi}+\Delta_{\xi}\left(\partial_{\theta_{n}}-\partial_{\phi}\right)\right] / \sqrt{2} .
$$

As one would expect, pseudocreators $b_{n}^{\dagger}$ increase the total excitation number in the difference-mode subspace by one. The number of pseudocreators, however, is $N$ and thus exceeds the number of difference-mode creation operators $a_{\mu}^{\dagger}$ by one. Indeed, the pseudocreators obey $\sum_{n} b_{n}=0$ and, hence, are not linearly dependent. They obey the nonstandard commutation relation

$$
\left[b_{m}, b_{n}^{\dagger}\right]=\delta_{m n}-1 / N .
$$

For the price of this anomalous commutator, we obtain operators that transform with elegant simplicity. Specifically, under array-variable permutations $\sigma \in \mathrm{S}_{N}$, the $b_{n}^{\dagger}$ operators simply undergo the following permutations:

$$
\sigma\left(b_{n}^{\dagger}\right)=\sum_{m} D(\sigma)_{n m} b_{m}^{\dagger}=b_{\sigma(n)}^{\dagger} .
$$

This simple transformation law will be crucial for finding the irreducible subspaces of $\mathcal{V}_{\mathrm{T}}$.

We next extend the language of difference-mode excitations to pseudomode excitations and define the states 


$$
|\mathbf{t}\rangle=\prod_{n=1}^{N}\left(b_{n}^{\dagger}\right)^{t_{n}}|\overrightarrow{0}\rangle \quad \text { (not normalized), }
$$

where the vector $\mathbf{t}=\left(t_{1}, t_{2}, \ldots, t_{N}\right)$ specifies the excitation numbers $t_{n} \in \mathbb{N}_{0}$ for each pseudomode $b_{n}^{\dagger}$. Using the inverse of Eq. (A6), $a_{\mu}^{\dagger}=\sum_{n} W_{\mu n} b_{n}^{\dagger}$, it is simple to confirm that

$$
\mathcal{V}_{\mathrm{T}}=\operatorname{span}\left\{|\mathbf{t}\rangle \mid \sum_{n} t_{n}=\mathrm{T}\right\}
$$

i.e., the pseudomode excitations span the difference-mode subspaces $\mathcal{V}_{\mathrm{T}}$ one by one. For example, the pseudomode states $b_{n}^{\dagger}|\overrightarrow{0}\rangle$ span the irreducible subspace $\mathcal{V}_{1}$ indexed by $(N-1,1)$ (see Fig. 3).

For a state with given pseudomode excitations $\mathbf{t}=$ $\left(t_{1}, t_{2}, \ldots, t_{N}\right)$, Eq. (A8) implies that $\sigma \in \mathrm{S}_{N}$ simply permutes the pseudomode-excitation numbers according to $\mathbf{t} \rightarrow \mathbf{t}^{\prime}=\left(t_{\sigma(1)}, t_{\sigma(2)}, \ldots, t_{\sigma(N)}\right)$.

Example: The state with three excitations in pseudomode $n=1$ and four excitations in pseudomode $n=2$ is $\mathbf{t}=(3,4,0, \ldots, 0)$. A permutation may transform it into $\mathbf{t}^{\prime}=(4,0,3,0, \ldots, 0)$, for example, but not into $(2,5,0, \ldots, 0)$, even though the latter state still has the same total excitation number $\mathrm{T}=7$.

For each state $|\mathbf{t}\rangle$ with $\mathrm{T}=\sum_{n} t_{n}$, we define the subspace spanned by itself and its permuted partner states as

$$
\mathcal{V}_{[\mathbf{t}]}:=\operatorname{span}\left\{\left|\mathbf{t}^{\prime}\right\rangle=|\sigma \mathbf{t}\rangle \mid \sigma \in \mathrm{S}_{N}\right\} \subset \mathcal{V}_{\mathrm{T}} .
$$

As suggested by the notation, [t] may be understood as an equivalence class when defining $\mathbf{t} \sim \mathbf{t}^{\prime}: \Leftrightarrow$ There exists a $\sigma \in \mathrm{S}_{N}$ such that $\mathbf{t}^{\prime}=\sigma \mathbf{t}$. By construction, the $\mathcal{V}_{[\mathbf{t}]}$ form $\mathrm{S}_{N}$-invariant subspaces, and their unions cover each $\mathcal{V}_{\mathrm{T}}$ :

$$
\mathcal{V}_{\mathrm{T}}=\bigcup_{\{[\mathrm{t}]} \bigcup_{\left.\mathrm{T}=\sum_{n} t_{n}\right\}} \mathcal{V}_{[\mathrm{t}]} .
$$

Note that, due to linear dependence of the pseudomodes, subspaces for inequivalent excitation classes $[\mathbf{t}] \cap\left[\mathbf{t}^{\prime}\right]=\varnothing$ may, nonetheless, have a nonzero intersection, $\mathcal{V}_{[\mathbf{t}]} \cap$ $\mathcal{V}_{\left[\mathrm{t}^{\prime}\right]} \neq \varnothing$.

We will first discuss the decomposition of $\mathcal{V}_{[\mathrm{t}]}$ into irreducible subspaces as if $b_{n}$ were orthogonal modes. In step 1, we thus temporarily drop the cautionary prefix "pseudo" and show that the basis vectors $|\mathbf{t}\rangle$ can then be relabeled in such a way to reveal isomorphism between $\mathcal{V}_{[t]}$ and the corresponding permutation module $M^{\lambda_{\mathrm{t}}}$. In step 2, we then utilize the important theorem for the decomposition of $M^{\lambda_{\mathrm{t}}}$ that identifies semistandard tableaux as the indexing set for all irreducible subspaces. In both steps, we introduce the necessary terminology and explain the construction. We do not provide proofs of the underlying theorems but instead refer the interested reader to Chapter 2 of Sagan's book [42]. Finally, in step 3, we return
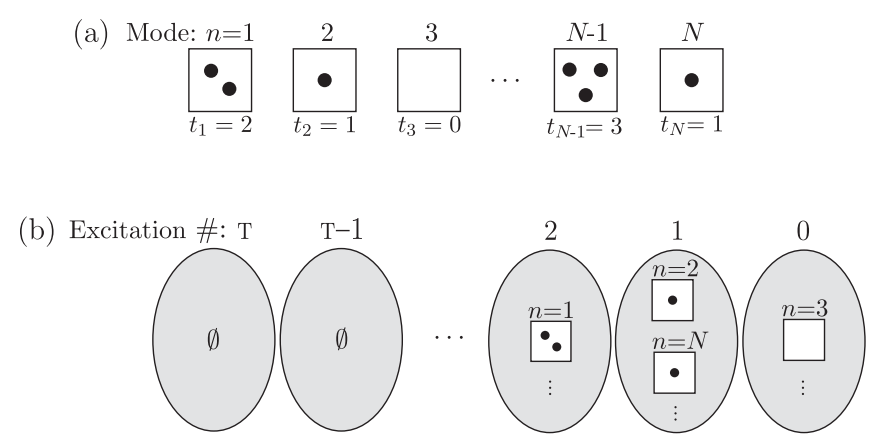

FIG. 6. Labeling schemes for difference-mode excitations. (a) The labels specify excitation numbers, mode by mode. (b) The labels specify mode indices, excitation number by excitation number.

to the issue of the linear dependence of $b_{n}$ modes and show how the usual construction can be modified to account for the linear dependence in a simple fashion.

\section{Step 1: Isomorphism between $\mathcal{V}_{[t]}$ and the permutation module $\boldsymbol{M}^{\boldsymbol{\lambda}_{\mathrm{t}}}$}

The excitation numbers $\mathbf{t}=\left(t_{1}, t_{2}, \ldots, t_{N}\right)$ label each state in $\mathcal{V}_{[t]}$ by specifying excitation numbers, mode by mode. An alternative labeling scheme (Fig. 6) consists of specifying mode numbers, excitation level by excitation level.

To specify the procedure of switching from the first labeling scheme to the second, we define the partition $\left(\lambda_{\mathbf{t}}\right)$ associated with $\mathbf{t}$ as follows. Consulting $\mathbf{t}$ and for each integer $f=0,1, \ldots, \mathrm{T}$, count

$$
\lambda_{f}^{\prime}=\text { (number of modes with } f \text { excitations). }
$$

The resulting sequence $\left(\lambda_{0}^{\prime}, \lambda_{1}^{\prime}, \ldots, \lambda_{\mathrm{T}}^{\prime}\right)$ sums to $N$, the total number of modes. By sorting entries in this sequence in decreasing order and dropping all 0 entries, we obtain the partition $\left(\lambda_{\mathbf{t}}\right)$ associated with $\mathbf{t}$. Excitation numbers $\mathbf{t}$ and $\mathbf{t}^{\prime}$ in the same equivalence class always have the same associated partition.

Example: For $N=4$ and excitation numbers $\mathbf{t}=$ $(1,3,0,1)$, one obtains the sequence $(1,2,0,1,0,0)$ and thus the associated partition $\left(\lambda_{\mathbf{t}}\right)=(2,1,1)$.

The partition $\left(\lambda_{\mathbf{t}}\right)=\left(\lambda_{1}, \lambda_{2}, \ldots, \lambda_{F}\right)$ is represented by a Young diagram, which is an array of squares where row $f$ has $\lambda_{f}$ squares. From the Young diagram $\left(\lambda_{\mathbf{t}}\right)$, we obtain the Young tableau $\Theta_{\mathbf{t}}$ associated with $\mathbf{t}$ by filling the boxes with the mode indices from 1 to $N$ in such a way that mode indices with the same excitation number appear in the same row.

Example: For $\mathbf{t}=(1,3,0,1)$, the Young diagram of $\left(\lambda_{\mathbf{t}}\right)=(2,1,1)$ and a Young tableau associated with $\mathbf{t}$ are given by 


$$
\left(\lambda_{\mathbf{t}}\right)=\square \quad \text { and } \Theta_{\mathbf{t}}=\begin{array}{l|l}
\hline & =4 \\
\hline &
\end{array} .
$$

From the sorting function used to order the entries in the partition $\left(\lambda_{\mathbf{t}}\right)$, one can infer which row in the tableau refers to which excitation number. As a result, the Young tableau lists the mode indices corresponding to each excitation number as intended. One additional modification is required to turn it into the desired state label.

For this modification, note that the transposition (interchange) of two mode indices with the same excitation number leads to a new tableau (consider an interchange of entries 1 and 4 in the previous example) but not to a new state $|\mathbf{t}\rangle$. To remove this ambiguity, we define two tableaux as row equivalent, $\Theta_{\mathbf{t}} \sim^{\mathrm{R}} \Theta_{\mathbf{t}}^{\prime}$ : $\Leftrightarrow$ Permutations of elements within each row can transform $\Theta_{\mathbf{t}}^{\prime}$ to $\Theta_{\mathbf{t}}$. The resulting equivalence class $\left[\Theta_{t}\right]$ is called the tabloid associated with $\mathbf{t}$ and serves as the new label for each state.

Example: Using $\mathbf{t}=(1,3,0,1)$ as above, the associated tabloid is

$$
\left[\Theta_{\mathbf{t}}\right]=\left\{\begin{array}{l|l|l|l}
1 & 4 & 4 & 1 \\
\hline 3 & , & 3 & \\
2 & & & 2
\end{array}\right\}
$$

It is useful to note that $\left[\Theta_{t}\right]$ can also be expressed as $\left[\Theta_{\mathbf{t}}\right]=\left\{\sigma \Theta_{\mathbf{t}} \mid \sigma \in \mathrm{R} \Theta_{\mathbf{t}}\right\}$, where $\mathrm{R}_{\mathbf{t}} \subset \mathrm{S}_{N}$ is the subset of permutations that only interchange entries in each row of the tableau $\Theta_{t}$. $R \Theta_{t}$ is called the row stabilizer of $\Theta_{t}$. Below, we will also encounter the column stabilizer $\mathrm{C}_{\mathrm{t}}$, with the analogous definition referring to columns instead of rows.

With these definitions and observations, we have established a one-to-one map $|\mathbf{t}\rangle \leftrightarrow\left|\left[\Theta_{\mathbf{t}}\right]\right\rangle$ that achieves the important goal of relating $\mathcal{V}_{[\mathrm{t}]}$ to a central object in the representation theory of $\mathrm{S}_{N}$ : the permutation module $M^{\lambda_{\mathrm{t}}}$ defined by

$$
M^{\lambda_{\mathbf{t}}}=\mathbb{C}\left\{\left[\Theta_{\mathbf{t}}\right] \mid \mathbf{t} \in[\mathbf{t}]\right\}
$$

Since each permutation module is defined in terms of tabloids, the group action for $\mathcal{V}_{[t]}$ and for $M^{\lambda_{t}}$ is easily verified to be identical, and the two vector spaces are hence isomorphic as $\mathrm{S}_{N}$ representations.

\section{Step 2: Decomposing $\mathcal{V}_{[\mathrm{t}]}$ and constructing basis vectors for all irreducible subspaces}

The great benefit of identifying $\mathcal{V}_{[\mathbf{t}]}$ as isomorphic to $M^{\lambda_{t}}$ lies in the availability of mathematical tools for decomposing the permutation modules into their irreducible subspaces (see Sagan [42], Sec. 2.10). For the symmetric group $S_{N}$, each irreducible representation $S^{\mu}$ is labeled uniquely by a partition $(\mu)$ of $N$. Consequently, the general decomposition takes the form

$$
M^{\lambda} \cong \bigoplus_{\mu} m_{\mu \lambda} S^{\mu}
$$

where $m_{\mu \lambda} \in \mathbb{N}_{0}$ is the multiplicity of the irreducible subspace $S^{\mu}$ within $M^{\lambda}$. For a given $\mathcal{V}_{[\mathrm{t}]} \simeq M^{\lambda_{\mathrm{t}}}$, we wish to obtain the basis vectors for each of the copies (if any) of $S^{\mu}$ contained in it. The basis vectors are obtained by means of semistandard tableaux, which we define next.

For the excitation numbers $\mathbf{t}$, an associated semistandard tableau $\Sigma_{t}^{\mu}$ is constructed from the Young diagram for $(\mu)$ [where $(\mu)$ need not be $\left(\lambda_{\mathbf{t}}\right)$ ] by filling its squares with the excitation numbers $t_{1}, t_{2}, \ldots, t_{N}$ in such a manner that entries in each row weakly increase $(\leq)$ and that entries in each column strictly increase $(<)$. Along with $\Sigma_{t}^{\mu}$, we consider standard tableaux $\Theta_{\mathrm{st}}^{\mu}$ of the same shape $(\mu)$ that are Young tableaux with entries increasing in each row and column. This way, we can set up a lookup function $\vartheta:\{1,2, \ldots, N\} \rightarrow\left\{t_{1}, t_{2}, \ldots, t_{N}\right\}$ that extracts the position of the integer $n$ in the reference tableau $\Theta_{\text {st }}^{\mu}$ and returns the excitation-number entry found in the semistandard tableau $\Sigma_{t}^{\mu}$ at the corresponding position. To keep the notation simple, we do not usually make explicit the dependence of $\vartheta$ on $\Sigma_{\mathrm{t}}^{\mu}$ and $\Theta_{\mathrm{st}}^{\mu}$.

Example: For $\mathbf{t}=(0,1,0,3)$ and partition $(\mu)=(2,2)$, an associated semistandard tableau and standard tableau are

$$
\Sigma_{\mathbf{t}}^{\mu}=\begin{array}{l|l}
0 & 0 \\
\hline 1 & 3
\end{array}, \quad \Theta_{\mathrm{st}}^{\mu}=\begin{array}{ll|}
1 & 3 \\
2 & 4
\end{array} .
$$

The lookup function then yields the following results:

\begin{tabular}{lllll}
$n$ & 1 & 2 & 3 & 4 \\
\hline$\vartheta(n)$ & 0 & 1 & 0 & 3
\end{tabular}.

With this preparation, one now obtains the basis states spanning the instance(s) of $S^{\mu}$ within $\mathcal{V}_{[\mathrm{t}]}$ from

$\left|\Sigma_{\mathbf{t}}^{\mu} ; \Theta_{\mathrm{st}}^{\mu}\right\rangle=\sum_{\sigma \in \mathrm{C} \Theta_{\mathrm{st}}^{\mu}} \sum_{\tau \in \mathrm{R} \Theta_{\mathrm{st}}^{\mu}} \operatorname{sgn}(\sigma) \sigma \tau\left[b_{1}^{\dagger \vartheta(1)} b_{2}^{\dagger \vartheta(2)} \cdots b_{N}^{\dagger \vartheta(N)}\right]|\overrightarrow{0}\rangle$,

where permutations $\sigma, \tau \in \mathrm{S}_{N}$ act on the mode indices, i.e., the subscripts of the $b_{n}^{\dagger}$ operators, as before. (Caveat: As defined above, the basis states are not yet normalized.) Each semistandard tableau $\Sigma_{t}^{\mu}$ yields an irreducible subspace

$$
\mathcal{V}_{\Sigma_{\mathbf{t}}^{\mu}}=\operatorname{span}\left\{\left|\Sigma_{\mathbf{t}}^{\mu} ; \Theta_{\mathrm{st}}^{\mu}\right\rangle \mid \text { all standard tableaux } \Theta_{\mathrm{st}}^{\mu}\right\} .
$$

By considering all possible partitions $(\mu)$ and associated semistandard tableaux $\Sigma_{\mathbf{t}}^{\mu}$, we thus completely decompose $\mathcal{V}_{[t]}$ into linearly independent irreducible subspaces. (The set of partitions one needs to consider can be restricted by considering the dominance ordering of partitions [42].) 


\section{Step 3: Linear dependence of $\boldsymbol{b}_{\boldsymbol{n}}$ modes and restricted semistandard tableaux}

In steps 1 and 2, we have ignored the linear dependence of $b_{n}$ expressed by the constraint $\sum_{n} b_{n}=0$. Once linear dependence is taken into account, the states from Eq. (A16) still span

$$
\mathcal{V}_{\mathrm{T}}=\operatorname{span}\left\{\left|\Sigma_{\mathbf{t}}^{\mu} ; \Theta_{\mathrm{st}}^{\mu}\right\rangle \mid \Sigma_{\mathbf{t}}^{\mu}, \Theta_{\mathrm{st}}^{\mu} \text { semi-standard and standard tableaux, } \mathrm{T}=\sum_{n} t_{n}\right\}
$$

However, in general, they are linearly dependent. Fortunately, the removal of this linear dependence can be achieved by a harmless modification of our previous procedure. This modification consists of an additional restriction on the set of admissible semistandard tableaux $\Sigma_{\mathbf{t}}^{\mu}$. Specifically, we will show that restricting the semistandard tableaux to the set

$$
\mathcal{R}_{\mathrm{T}}=\left\{\Sigma_{\mathbf{t}}^{\mu} \mid \Sigma_{\mathbf{t}}^{\mu} \text { has no"1"in its first row, } \sum_{n} t_{n}=\mathrm{T}\right\}
$$

removes the linear dependence and that

$$
\mathcal{B}_{\mathrm{T}}=\left\{\left|\Sigma_{\mathbf{t}}^{\mu} ; \Theta_{\mathrm{st}}^{\mu}\right\rangle \mid \Sigma_{\mathbf{t}}^{\mu} \in \mathcal{R}_{\mathrm{T}}, \Theta_{\mathrm{st}}^{\mu} \text { standard tableau }\right\}
$$

forms a basis for $\mathcal{V}_{\mathrm{T}}$. Furthermore, each subspace spanned by states with a fixed restricted semistandard tableau $\Sigma_{t}^{\mu}$ retains its character as an irreducible representation indexed by the partition $(\mu)$.

To prove this assertion, we first show that every state $\left|\Sigma_{\mathrm{t}}^{\mu \prime} ; \Theta_{\mathrm{st}}^{\mu}\right\rangle$ obtained for a "forbidden" semistandard tableau $\Sigma_{\mathbf{t}}^{\mu \prime} \notin \mathcal{R}_{t}$ can be written as a linear combination of states $\left|\Sigma_{\mathbf{t}}^{\mu} ; \Theta_{\mathrm{st}}^{\mu}\right\rangle$ from the restricted set, i.e., $\Sigma_{\mathrm{t}}^{\mu} \in \mathcal{R}_{\mathrm{T}}$. Consider the vectors constructed in Eq. (A16) and note that the row stabilizer can always be separated into the stabilizer of only the first row $R_{1}$ and the stabilizer of all remaining rows $R^{\prime}$ :

$$
\sum_{\tau \in \mathrm{R} \Theta_{\mathrm{st}}^{\mu}} \tau=\sum_{\tau^{\prime} \in \mathrm{R}^{\prime}} \tau^{\prime} \circ \sum_{\tau \in \mathrm{R}_{1}} \tau .
$$

Example: In this example and all that follow, we consider the forbidden state vector

$$
\left.\begin{array}{|l|l|l|l|l|l|}
\hline 0 & 1 & 2 \\
\hline 1 & 4 & ; & 3 & 3 & 5 \\
\hline 2 & 4 &
\end{array}\right\rangle .
$$

The stabilizer for row 1 consists of $R_{1}=$ $\{e,(13),(15),(35),(135),(153)\}$; the stabilizer for the remaining rows is $\mathrm{R}^{\prime}=\{e,(24)\}$.

Proceeding with the decomposition of the forbidden state vector $\left|\Sigma_{\mathbf{t}}^{\mu \prime} ; \Theta_{\mathrm{st}}^{\mu}\right\rangle$ in terms of states with restricted semistandard tableaux, let $M=\left\{m_{1}, m_{2}, \ldots, m_{N}\right\}$ denote the entries of the standard tableau $\Theta_{\mathrm{st}}^{\mu}$ (reading left to right, row by row) and let $M_{1}=\left\{m_{1}, m_{2}, \ldots, m_{r}\right\}$ denote the entries in row 1 only. Similarly, let $\left(t_{1}, t_{2}, \ldots, t_{r}\right)$ be the integer excitation numbers in the first row of the semistandard tableau $\Sigma_{t}^{\mu \prime}$ and (without loss of generality) assume that $t_{1}=\cdots=t_{q-1}=0$ and $t_{q}=1$ for $q \leq r$.

Next, we introduce the sets $\Lambda_{m}=\left\{n_{r}, n_{r-1}, \ldots, n_{m}\right\}$ and rewrite the stabilizer of row 1 as

$$
\begin{aligned}
& \sum_{\tau \in \mathrm{R}_{1}} \tau\left[b_{m_{q}}^{\dagger} b_{m_{q+1}}^{\dagger t_{q+1}} \cdots b_{m_{r}}^{\dagger t_{r}}\right] \\
& \quad=(q-1) ! \sum_{n_{r} \in M_{1}} b_{n_{r}}^{\dagger t_{r}} \sum_{n_{r-1} \in M_{1} \backslash \Lambda_{r}} b_{n_{r-1}}^{\dagger t_{r-1}} \cdots \sum_{n_{q} \in M_{1} \backslash \Lambda_{q+1}} b_{n_{q}}^{\dagger} .
\end{aligned}
$$

By construction, each successive sum over pseudomode indices $n_{r}, n_{r-1}, \ldots$ is associated with weakly decreasing excitation numbers $t_{r} \geq t_{r-1} \geq \cdots$, and the final sum over pseudomode indices $n_{q}$ corresponds to the case of a single excitation (entry " 1 " in row 1 ).

Example: Continuing with our previous example, the above equality takes the form

$$
\begin{aligned}
\sum_{\tau \in \mathrm{R}_{1}} \tau\left(b_{1}^{\dagger 0} b_{3}^{\dagger} b_{5}^{\dagger 2}\right)= & \sum_{n_{3} \in\{1,3,5\}} b_{n_{3}}^{\dagger 2} \sum_{n_{2} \in\{1,3,5\} \backslash\left\{n_{3}\right\}} b_{n_{2}}^{\dagger} \\
= & b_{1}^{\dagger 2}\left(b_{3}^{\dagger}+b_{5}^{\dagger}\right)+b_{3}^{\dagger 2}\left(b_{1}^{\dagger}+b_{5}^{\dagger}\right) \\
& +b_{5}^{\dagger 2}\left(b_{3}^{\dagger}+b_{1}^{\dagger}\right) .
\end{aligned}
$$

Next, we use the linear dependence of the pseudomodes to rewrite the final sum in Eq. (A23) as

$$
\sum_{n_{q} \in M_{1} \backslash \Lambda_{q+1}} b_{n_{q}}^{\dagger}=-\sum_{n \in \Lambda_{q+1}} b_{n}^{\dagger}-\sum_{n \in M \backslash M_{1}} b_{n}^{\dagger} .
$$

The transformed expression has two separate sums over $n$ : a sum over pseudomodes that, according to Eq. (A23), are already occupied and a sum over pseudomodes in rows $2,3, \ldots$ of the standard tableau. The increase of excitation number produced by Eq. (A24) hence only affects pseudomodes that are already occupied.

Example: Again continuing with our previous example, we find that 
$\sum_{n_{2} \in\{1,3,5\} \backslash\left\{n_{3}\right\}} b_{n_{2}}^{\dagger}=-\sum_{n \in\left\{n_{3}\right\}} b_{n}^{\dagger}-\sum_{n \in\{2,4\}} b_{n}^{\dagger}$.

Finally, we inspect the full state vector by using Eqs. (A21), (A23), and (A24). The resulting terms associated with a single index $n$ [Eq. (A24)] can be reexpressed as a sum over the complete row stabilizer $\mathrm{R}$ and associated with a tableau where entry " 1 " in the first row of $\Sigma_{t}^{\mu \prime}$ has been eliminated and another entry $1 \leq t_{n}$ of $\Sigma_{\mathrm{t}}^{\mu \prime}$ has been increased by one: In such a way, the state with a forbidden semistandard tableau

\begin{tabular}{|c|c|c|c|c|c|c|}
\hline 0 & $\cdots$ & 0 & 1 & $t_{q+1}$ & $\cdots$ & $t_{r}$ \\
\hline$t_{r+1}$ & $\cdots$ & $t_{r_{2}}$ & \multicolumn{1}{l}{} \\
\hline$\vdots$ & \multicolumn{1}{|l}{} \\
\cline { 1 - 3 } &
\end{tabular}

is decomposed into a linear combination of states with tableaux

\begin{tabular}{|c|c|c|c|c|c|c|}
\hline 0 & $\cdots$ & 0 & $\mathbf{0}$ & $t_{q+1}+1$ & $\cdots$ & $t_{r}$ \\
\hline$t_{r+1}$ & $\cdots$ & $t_{r_{2}}$ & \multicolumn{1}{|l}{} \\
\hline$\vdots$ & \multicolumn{1}{|l}{}
\end{tabular}

\begin{tabular}{|c|c|c|c|c|c|c|}
\hline 0 & $\cdots$ & 0 & 0 & $t_{q+1}$ & $\cdots$ & $t_{r}+1$ \\
\hline$t_{r+1}$ & $\cdots$ & $t_{r_{2}}$ & \multicolumn{1}{l}{} \\
\hline$\vdots$ & \multicolumn{1}{|l}{} \\
\cline { 1 - 2 } &
\end{tabular}

\begin{tabular}{|c|c|c|c|c|c|c|}
\hline 0 & $\cdots$ & 0 & 0 & $t_{q+1}$ & $\cdots$ & $t_{r}$ \\
\hline$t_{r+1}+1$ & $\cdots$ & $t_{r_{2}}$ & \multicolumn{1}{|l}{} \\
\hline$\vdots$ & \multicolumn{1}{|l}{} \\
\cline { 1 - 2 } & & \multicolumn{1}{|l}{}
\end{tabular}

\begin{tabular}{|c|c|c|c|c|c|c|}
\hline 0 & $\cdots$ & 0 & 0 & $t_{q+1}$ & $\cdots$ & $t_{r}$ \\
\hline$t_{r+1}$ & $\cdots$ & $t_{r_{2}}+1$ & \multicolumn{1}{l}{} \\
\hline$\vdots$ & \multicolumn{1}{|l}{} \\
\cline { 1 - 2 } &
\end{tabular}

where we have used bold red text to emphasize the changes relative to the forbidden tableau. In cases where the above procedure results in a tableau that is not semistandard, a straightening algorithm can be applied to generate the corresponding semistandard tableau (see [42], Sec. 2.6). Importantly, the straightening algorithm does not change the content of the tableaux and thus does not change the fact that our procedure expresses the forbidden tableau in terms of a semistandard tableau with fewer " 1 " entries in row 1 . Using this procedure, repeatedly if necessary, we can decompose any state with a forbidden semistandard tableau as claimed. It is important, of course, that the removal of states occurs at the level of entire subspaces (indexed by the forbidden semistandard tableaux) while the group action and hence the irreducibility of the remaining subspaces is unharmed.

Example: We complete our running example by decomposing the forbidden state vector

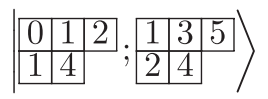

in the restricted basis. Following our previous steps, the state vector can be expressed as

$$
\begin{aligned}
= & \sum_{\sigma \in \mathrm{C}} \sum_{\tau \in \mathrm{R}^{\prime}} \operatorname{sgn}(\sigma) \sigma \tau\left[b _ { 2 } ^ { \dagger } b _ { 4 } ^ { \dagger 4 } \left(-\sum_{n_{3} \in M_{1}} b_{n_{3}}^{\dagger 3}\right.\right. \\
& \left.\left.-\sum_{n_{3} \in M_{1}} b_{n_{3}}^{\dagger 2} \sum_{n \in\{2,4\}} b_{n}^{\dagger}\right)\right] .
\end{aligned}
$$

Together with a combinatorial factor (here, $1 / 2$ ), the sums over $n_{3}$ and $\tau^{\prime} \in \mathrm{R}^{\prime}$ can be recombined into the full row stabilizer:

$$
\begin{aligned}
= & -\frac{1}{2} \sum_{\sigma \in \mathrm{C}} \sum_{\tau \in \mathrm{R}} \operatorname{sgn}(\sigma) \sigma \tau\left[b_{2}^{\dagger} b_{4}^{\dagger 4}\left(b_{5}^{\dagger 3}+b_{5}^{\dagger 2} \sum_{n \in\{2,4\}} b_{n}^{\dagger}\right)\right] \\
= & -\frac{1}{2} \sum_{\sigma \in \mathrm{C}} \sum_{\tau \in \mathrm{R}} \operatorname{sgn}(\sigma) \sigma \tau\left[b_{2}^{\dagger} b_{4}^{\dagger 4} b_{5}^{\dagger 3}+b_{2}^{\dagger 2} b_{4}^{\dagger 4} b_{5}^{\dagger 2}\right. \\
& \left.+b_{2}^{\dagger} b_{4}^{\dagger 5} b_{5}^{\dagger 2}\right] .
\end{aligned}
$$

We have thus completed our goal of expressing the original forbidden state vector as a linear combination of the "restricted" state vectors

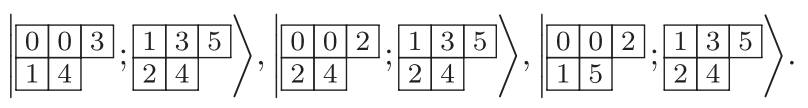

With the decomposition of forbidden semistandard tableaux in hand, we conclude by showing that the states $\left|\Sigma_{\mathrm{t}}^{\mu} ; \Theta_{\mathrm{st}}^{\mu}\right\rangle$ with restricted $\Sigma_{\mathrm{t}}^{\mu} \in \mathcal{R}_{\mathrm{t}}^{\mu}$ not only span each $V_{\mathrm{T}}$ but are linearly independent. The proof is based on a simple dimensional argument. Counting the number of basis elements with $\mathrm{T}$ excitations, we find

$$
\operatorname{dim} \mathcal{V}_{\mathrm{T}}=\frac{(N-2+\mathrm{T}) !}{\mathrm{T} !(N-2) !}
$$

This expression should be compared with the dimensionality of subspaces constructed with restricted semistandard tableaux $\Sigma_{\mathrm{t}}^{\mu} \in \mathcal{R}_{\mathrm{T}}$. For $\sum_{n} t_{n}=\mathrm{T} \leq 5$, the explicit listing of restricted semistandard tableaux is given in Table III. 
TABLE III. Irreducible subspaces for difference modes with total excitation number $\mathrm{T} \leq 5$. In this Table, the equivalence class [t] with $\lambda_{f}^{\prime}$ pseudomodes with exactly $f$ excitations is denoted $0^{\lambda_{0}^{\prime}} 1_{1}^{\lambda_{1}^{\prime}} \ldots$, where all entries with $\lambda_{f}^{\prime}=0$ are suppressed. All semistandard tableaux of shape $(\mu)$ and content $[\mathbf{t}]$ are listed in the corresponding row and column.

\begin{tabular}{|c|c|c|c|c|c|c|c|c|c|c|}
\hline $\mathrm{T}$ & {$[\mathbf{t}]=0^{\lambda_{0}^{\prime}} 1^{\lambda_{1}^{\prime}} \ldots$} & $(\mu)=(N)$ & $(N-1,1)$ & $(N-2,2)$ & $\left(N-2,1^{2}\right)$ & $(N-3,3)$ & $(N-3,2,1)$ & $(N-4,4)$ & $(N-4,3,1)$ & $(N-5,5)$ \\
\hline 0 & $0^{N}$ & \begin{tabular}{|l|l|l|}
0 & $\ldots$ & 0 \\
\end{tabular} & & & & & & & & \\
\hline 1 & $0^{N-1} 1$ & & \begin{tabular}{|l|l|}
0 & $\ldots$ \\
1 & \\
\end{tabular} & & & & & & & \\
\hline \multirow[t]{2}{*}{2} & $0^{N-1} 2$ & \begin{tabular}{|l|l|l|l|}
0 & 0 & $\ldots$ & 0 \\
\end{tabular} & \begin{tabular}{|l|l|l|}
0 & $\cdots$ & 0 \\
2 & \\
\end{tabular} & & & & & & & \\
\hline & $0^{N-2} 1^{2}$ & & & \begin{tabular}{|l|l|l|}
0 & $\ldots$ & 0 \\
1 & 1 & \\
\end{tabular} & & & & & & \\
\hline \multirow[t]{3}{*}{3} & $0^{N-1} 3$ & \begin{tabular}{|l|l|l|l|}
0 & $\ldots$ & 0 & 3 \\
\end{tabular} & \begin{tabular}{|l|l|}
0 & $\ldots .0$ \\
3 &
\end{tabular} & & & & & & & \\
\hline & $0^{N-2} 12$ & & \begin{tabular}{|l|l|l|l|}
0 & $\ldots$ & 0 \\
1
\end{tabular} & \begin{tabular}{|l|l|}
0 & $\ldots$ \\
1 & 2 \\
\end{tabular} & \begin{tabular}{|l|l|l|}
0 & $\ldots$ & 0 \\
1 & \\
2 & \\
\end{tabular} & & & & & \\
\hline & $0^{N-3} 1^{3}$ & & & & & \begin{tabular}{|l|l|l|l|}
0 & 0 & $\ldots$ & 0 \\
1 & 1 & 1 \\
\end{tabular} & & & & \\
\hline \multirow[t]{5}{*}{4} & $0^{N-1} 4$ & \begin{tabular}{|l|l|l|l|}
0 & $\ldots$ & 0 & 4 \\
\end{tabular} & \begin{tabular}{|l|l|}
0 & $\cdots$ \\
4 & \\
\end{tabular} & & & & & & & \\
\hline & $0^{N-2} 13$ & & \begin{tabular}{|l|l|l|l|}
0 & $\ldots$ & 0 & \\
1
\end{tabular} & \begin{tabular}{|l|l|}
0 & $\ldots$ \\
1 & 3 \\
\end{tabular} & \begin{tabular}{|l|l|l|}
0 & $\ldots$ & 0 \\
1 & & \\
\cline { 1 - 1 } 3 & &
\end{tabular} & & & & & \\
\hline & $0^{N-2} 2^{2}$ & \begin{tabular}{|l|l|l|l|}
0 & $\ldots$ & 0 & 2 \\
\end{tabular} & 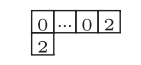 & \begin{tabular}{|l|l|}
0 & $\ldots$ \\
2 & 2 \\
\end{tabular} & & & & & & \\
\hline & $0^{N-3} 1^{2} 2$ & & & \begin{tabular}{|l|l|l|l|}
0 & $\ldots$ & 0 & 2 \\
1 & 1 & & \\
\end{tabular} & & \begin{tabular}{|l|l|l|l|}
0 & 0 & $\ldots$ & 0 \\
1 & 1 & 2 \\
\end{tabular} & \begin{tabular}{|l|l|l|}
0 & $\ldots$ & 0 \\
1 & 1 & \\
2 & &
\end{tabular} & & & \\
\hline & $0^{N-4} 1^{4}$ & & & & & & & \begin{tabular}{|l|l|l|l|}
0 & 0 & 0 & $\ldots$ \\
1 & 1 & 1 & 1 \\
\end{tabular} & & \\
\hline \multirow[t]{8}{*}{5} & $0^{N-1} 5$ & \begin{tabular}{|l|l|l|}
0 & $\ldots$ & 0 \\
\end{tabular} & \begin{tabular}{|l|l|}
0 & $\cdots$ \\
5
\end{tabular} & & & & & & & \\
\hline & $0^{N-2} 14$ & & \begin{tabular}{|l|l|l|l|}
0 & $\ldots$ & 0 & 4 \\
1 & & \\
\end{tabular} & \begin{tabular}{|l|l|}
0 & $\ldots$. \\
1 & 4 \\
\end{tabular} & \begin{tabular}{|l|l|l|}
0 & $\ldots$ & 0 \\
1 & \\
4 &
\end{tabular} & & & & & \\
\hline & $0^{N-2} 23$ & 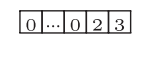 & \begin{tabular}{|l|l|l|l|l|l|l|l|}
0 & $\ldots$ \\
2
\end{tabular} & \begin{tabular}{|l|l|l|}
0 & $\ldots$ & 0 \\
2 & 3 & \\
\end{tabular} & \begin{tabular}{|l|l|}
$0 . \ldots 10$ \\
2
\end{tabular} & & & & & \\
\hline & & & \begin{tabular}{|l|l|l|l|}
0 & $\ldots$ & 0 & \\
3 &
\end{tabular} & & & & & & & \\
\hline & $0^{N-3} 1^{2} 3$ & & & \begin{tabular}{|l|l|l|l|}
0 & $\ldots$ & 0 & 3 \\
1 & 1 & \\
\end{tabular} & & \begin{tabular}{|l|l|l|l|}
0 & 0 & $\ldots$ & 0 \\
1 & 1 & 3 & \\
\end{tabular} & \begin{tabular}{|l|l|l|}
0 & $\ldots$ & 0 \\
1 & 1 & \\
3 & &
\end{tabular} & & & \\
\hline & $0^{N-3} 12^{2}$ & & \begin{tabular}{|l|l|l|l|l|}
0 & $\cdots$ & 0 & 2 & 2 \\
1 & & \\
\end{tabular} & \begin{tabular}{|l|l|l|l|}
0 & $\ldots$ & 0 & 2 \\
1 & 2 & & \\
\end{tabular} & \begin{tabular}{|l|l|l|l|}
0 & $\ldots$ & 0 & 2 \\
1 & & & \\
2 & & & \\
$y$
\end{tabular} & \begin{tabular}{|l|l|l|l|}
0 & 0 & $\ldots$ & 0 \\
1 & 2 & 2 \\
\end{tabular} & \begin{tabular}{|l|l|l|}
0 & $\ldots$ & 0 \\
1 & 2 & \\
2 & &
\end{tabular} & & & \\
\hline & $0^{N-4} 1^{3} 2$ & & & & & 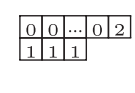 & & \begin{tabular}{|l|l|l|l|l|}
0 & 0 & 0 & $\ldots$ & 0 \\
1 & 1 & 1 & 2 \\
\end{tabular} & \begin{tabular}{|l|l|l|l|}
0 & 0 & $\ldots$ & 0 \\
\cline { 1 - 3 } & 1 & 1 & \\
\cline { 1 - 3 } 2 & \multicolumn{3}{|c}{} \\
\end{tabular} & \\
\hline & $0^{N-5} 1^{5}$ & & & & & & & & & \begin{tabular}{|l|l|l|l|l|}
0 & 0 & 0 & 0 & $\ldots$ \\
1 & 1 & 1 & 1 & 0 \\
\end{tabular} \\
\hline
\end{tabular}


For comparison with Eq. (A28), note that the dimension $d_{\mu}$ of the irreducible representation of $S_{N}$ indexed by partition $(\mu)$ can be obtained by the hook-length formula; see Sagan [42], Sec. 3.10. We have verified that

$$
\sum_{\Sigma_{\mathrm{t}}^{\mu} \in \mathcal{R}_{\mathrm{T}}} d_{\mu}=\frac{(N-2+\mathrm{T}) !}{\mathrm{T} !(N-2) !}
$$

for all $\mathrm{T} \leq 5$ and leave it as a conjecture that equality continues to hold for all higher $\mathrm{T}$.

Example: As an example, we consider $\mathrm{T}=2$. Using the hook-length formula, the sum of the dimension of the irreducible subspaces indexed by the semistandard tableaux

$$
\begin{array}{|l|l|l|l|l|l|l|l|l|l|}
\hline 0 & \cdots & 0 & 2 \\
\hline & \cdots & \cdots & 0 \\
\hline 2 & &
\end{array}, \begin{array}{|l|l|l|}
\hline & \cdots & 0 \\
\hline 1 & 1 & \\
\hline
\end{array}
$$

is equal to $1+N-1+N(N-3) / 2$. Simple arithmetic shows that this expression is equal to $\operatorname{dim} \mathcal{V}_{\mathrm{T}=2}=$ $N(N-1) / 2$ as expected.

In summary, the subspaces indexed by restricted semistandard tableaux decompose each $\mathcal{V}_{\mathrm{T}}$ into its irreducible subspaces. As a final remark, we note that, for $\mathrm{T}>2$, multiplicities of irreducible representations can exceed 1 . (Through $\mathrm{T}=5$, the largest multiplicity that occurs is 5 ; see Table III.) In such cases, the usual Gram-Schmidt procedure may be employed to generate orthogonal irreducible subspaces.

\section{APPENDIX B: CALCULATION OF PERTURBATIVE SHIFTS ACCORDING TO IRREDUCIBLE SUBSPACES}

We next discuss the calculation of first-order shifts of energy levels in each irreducible subspace under the $\mathrm{S}_{N}$ symmetric perturbation $\delta H_{U}$. In general, irreducible subspaces for the difference modes are labeled by restricted semistandard tableaux. [For $\mathrm{T} \leq 2$, it is sufficient to specify $\mathrm{T}$ and the partition $(\lambda)$ instead of full-blown semistandard tableaux. In this Appendix, we continue to employ the restricted semistandard tableau notation.]

To calculate the first-order shifts, we choose a unique element from each subspace. This selection is made by fixing a reference standard tableau $\Theta_{\text {ref }}^{\lambda}$, which we choose as the standard Young tableau of shape $(\lambda)$ with entries 1 through $N$ filled in column by column. Using this reference tableau, we obtain one representative state in each irreducible difference-mode subspace, which we denote by $\left|\Sigma_{\mathbf{t}}^{\lambda}\right\rangle$ $\left(\Sigma_{\mathbf{t}}^{\lambda} \in \mathcal{R}_{\mathbf{t}}^{\lambda}\right)$.

Example: The reference standard tableau for the partition $(\lambda)=(N-2,2)$ is

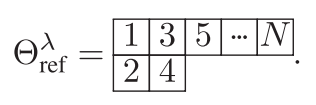

The state acting as the representative for the $\mathrm{T}=2,(\lambda)=$ $(N-2,2)$ subspace is then given by

$$
\begin{aligned}
& \left.\begin{array}{|l|l|l|l|l}
\hline 0 & 0 & 0 & \cdots & 0 \\
\hline 1 & 1 & &
\end{array}\right\rangle \sim \sum_{\sigma \in \mathrm{C}} \sum_{\tau \in \mathrm{R}} \operatorname{sgn}(\sigma) \sigma \tau\left(b_{2}^{\dagger} b_{4}^{\dagger}\right)|\overrightarrow{0}\rangle \\
& \sim\left(b_{2}^{\dagger}-b_{1}^{\dagger}\right)\left(b_{4}^{\dagger}-b_{3}^{\dagger}\right)|\overrightarrow{0}\rangle,
\end{aligned}
$$

where the column and row stabilizers $\mathrm{C}$ and $\mathrm{R}$ are defined with respect to the reference standard tableau. With Eq. (A7), we obtain

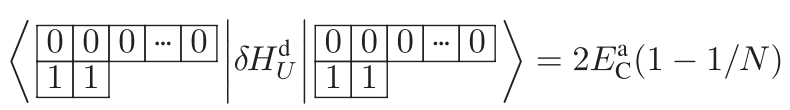

for the first-order shift of the irreducible subspace due to the effect of anharmonicity.

\section{APPENDIX C: LAGRANGIAN FOR SUPERINDUCTANCE AND DIFFERENCE-MODE VARIABLES}

After transforming to superinductance and differencemode variables, the Lagrangian of Eq. (1) can be cast into the form

$$
\begin{aligned}
\mathcal{L}_{\mathrm{SFM}}= & \frac{\hbar^{2}}{16 E_{\mathrm{C}}^{\mathrm{si}}} \dot{\phi}^{2}+\frac{\hbar^{2}}{16 E_{\mathrm{C}}^{\mathrm{a}}} \sum_{\mu} \dot{\xi}_{\mu}^{2}+E_{\mathrm{J}}^{\mathrm{b}} \cos \left(\phi+\varphi_{\mathrm{ext}}\right) \\
& +E_{\mathrm{J}}^{\mathrm{a}} \sum_{m} \cos \left[\phi / N+\sum_{\mu} W_{\mu m} \xi_{\mu}\right] .
\end{aligned}
$$

This expression for the Lagrangian has important advantages over its equivalent form expressed in terms of $\theta_{m}$. First, the kinetic energy is now diagonal. Second, lowenergy minima of the potential energy $U$ are in locations where each difference-mode variable vanishes, $\xi_{\mu}=0$. Third, fluctuations between minima are dominantly described by the $\phi$ variable. The ability to simultaneously expand around $\xi_{\mu}=0$ for each local minimum of $U$ is key in the derivation of the superinductance model that was previously used $[8,9]$.

\section{APPENDIX D: INCORPORATING CAPACITANCES TO GROUND}

The $(N+1)$ node variables $\varphi_{j}$ can be expressed in terms of the $N$ junction variables $\theta_{m}$ when using the constraint that the total charge $\sum_{j} n_{j}$ of all superconducting islands must be zero. To show that this statement is indeed true, we may use $\tau=\varphi_{0}$ as a reference variable and express every other node variable $\varphi_{j}(j=1, \ldots, N)$ as

$$
\varphi_{j}=\tau-\frac{j}{N} \varphi_{\mathrm{ext}}+\sum_{m=1}^{j} \theta_{m} .
$$

Note that $\dot{\tau} \Phi_{0} / 2 \pi$ represents a uniform voltage shift of all superconducting islands relative to ground and that $\tau$ is cyclic; i.e., the Lagrangian is independent of $\tau$. Hence, its conjugate momentum is conserved: $\partial \mathcal{L} / \partial \dot{\tau}=$ const. This constant of motion, in fact, corresponds to the total charge $n_{\text {tot }}$ since 


$$
\frac{\partial \mathcal{L}}{\partial \dot{\tau}}=\sum_{j} \frac{\partial \varphi_{j}}{\partial \tau} \frac{\partial \mathcal{L}}{\partial \dot{\varphi}_{j}}=\sum_{j} n_{j}=n_{\text {tot }}
$$

Imposing the constraint $n_{\text {tot }}=0$ thus allows us to eliminate $\dot{\tau}$ from the Lagrangian and to work with a Lagrangian (strictly speaking, a Routhian) that only depends on $\theta_{m}$ and $\dot{\theta}_{m}$. Using this procedure, the contribution to the kinetic energy due to ground capacitances $\frac{1}{2}\left(\Phi_{0} / 2 \pi\right)^{2} \times$ $\sum_{i=0}^{N} C_{\mathrm{g} i} \dot{\varphi}_{i}^{2}$ in Eq. (1) takes the form $\frac{1}{2} \sum_{m n} \mathcal{G}_{m n} \dot{\theta}_{m} \dot{\theta}_{n}$, where

$$
G_{m n}=\frac{\left(\Phi_{0} / 2 \pi\right)^{2}}{\sum_{i=0}^{N} C_{\mathrm{g} i}} \sum_{i=0}^{\min \{m, n\}-1} \sum_{j=\max \{m, n\}}^{N} C_{\mathrm{g} i} C_{\mathrm{g} j} .
$$

Then, assuming that the two large superconducting islands surrounding the black-sheep junction have ground capacitance $C_{\mathrm{g}}^{\mathrm{b}}=e^{2} / 2 E_{\mathrm{g}}^{\mathrm{b}}$ while the ground capacitance of the remaining small array islands is $C_{\mathrm{g}}^{\mathrm{a}}=e^{2} / 2 E_{\mathrm{g}}^{\mathrm{a}}$ and using the variables $\xi_{\mu}$ defined in Eq. (3), the kinetic-energy terms of the Lagrangian [Eq. (1)] take the form

$$
\frac{\hbar^{2}}{16} \sum_{\mu, \nu=0}^{N-1}\left(M_{\mu \nu}+G_{\mu \nu}\right) \dot{\xi}_{\mu} \dot{\xi}_{\nu}
$$

Here, for compactness, we use the shorthand $\xi_{0}=\phi$. The symmetric $N \times N$ matrices $M$ and $G$ have the following form:

$M_{00}=1 / E_{\mathrm{C}}^{\mathrm{b}}+1 /\left(N E_{\mathrm{C}}^{\mathrm{a}}\right), \quad M_{0 \mu}=0, \quad M_{\mu \nu}=\delta_{\mu \nu} / E_{\mathrm{C}}^{\mathrm{a}}$

and

$$
\begin{aligned}
G_{00} & =\frac{1}{2 E_{\mathrm{g}}^{\mathrm{b}}}+\frac{(N-1)(N-2)}{12 N E_{\mathrm{g}}^{\mathrm{a}}}, \quad G_{0 \mu}=-\frac{o_{\mu+1} \mathrm{c}(\mu)}{2 E_{\mathrm{g}}^{\mathrm{a}} \sqrt{2 N} \mathrm{~s}(\mu)^{2}}, \\
G_{\mu \nu} & =\frac{\delta_{\mu \nu}}{4 E_{\mathrm{g}}^{\mathrm{a}} \mathrm{s}(\mu)^{2}}-\frac{E_{\mathrm{g}}^{\mathrm{b}} o_{\mu} o_{\nu} \mathrm{c}(\mu) \mathrm{c}(\nu)}{2 N E_{\mathrm{g}}^{\mathrm{a} 2}\left[2+(N-1) E_{\mathrm{g}}^{\mathrm{b}} / E_{\mathrm{g}}^{\mathrm{a}}\right] \mathrm{s}(\mu)^{2} \mathrm{~s}(\nu)^{2}},
\end{aligned}
$$

where $\mathrm{c}(\mu)$ and $\mathrm{s}(\mu)$ are shorthand for $\cos (\pi \mu / 2 N)$ and $\sin (\pi \mu / 2 N)$, respectively. Furthermore, the coefficient $o_{\mu}$ is 1 whenever the index $\mu$ is an odd integer and is zero otherwise.

Performing the Legendre transform, the perturbation from ground capacitances takes the form

$$
\delta H_{\mathrm{C}}=-4 \sum_{\mu, \nu=0}^{N-1}\left[(M+G)^{-1}-M^{-1}\right]_{\mu \nu} \partial_{\xi_{\mu}} \partial_{\xi_{\nu}} .
$$

For small ground capacitances, the entries of $G$ are small compared to those of $M$, and we approximate $(M+G)^{-1} \simeq M^{-1}-M^{-1} G M^{-1}$, which yields Eq. (9) in the main text.

\section{APPENDIX E: LIMITS OF THE PERTURBATIVE APPROACH}

Corrections in the main text are treated perturbatively, and we briefly comment on the necessary conditions for this approach to be valid. First, we remark that the energy scales $\delta E_{\mathrm{J}}^{\mathrm{sd}}$ and $\delta E_{\mathrm{C}}^{\mathrm{sd}}$ of Eqs. (13) and (14) must remain sufficiently small relative to the typical energy scales of the superinductance spectrum. Second, when the magnitude of $\left\langle\overrightarrow{0}\left|\delta H_{U}^{\mathrm{d}}\right| 2(N)\right\rangle$ or $\left\langle\overrightarrow{0}\left|\delta H_{\mathrm{C}}^{\mathrm{d}}\left(a_{1}^{\dagger}\right)^{2}\right| \overrightarrow{0}\right\rangle$ becomes of order $2 \Omega$, the ground state of the difference modes will require nonperturbative corrections. To prevent this breakdown of the perturbative approach, the respective inequalities

$$
\sqrt{N / 2} \ll 16 / \Delta_{\xi}^{2}, \quad\left(N \Delta_{\xi} / 2 \pi\right)^{2} \ll E_{\mathrm{g}}^{\mathrm{a}} / E_{\mathrm{C}}^{\mathrm{a}}
$$

must hold. Thus, to connect with the $N \rightarrow \infty$ limit (see, e.g., Ref. [39]), a different approximation scheme to model the low-energy spectrum of fluxonium may become necessary. However, in the case of the fluxonium samples previously studied in Refs. [8,9], the range of validity of the perturbative approach is well satisfied.

\section{APPENDIX F: PARAMETER VALUES USED IN NUMERICAL CALCULATIONS}

The specific parameters used in all calculations in the main text are $N=43, E_{\mathrm{C}}^{\mathrm{a}}=1.0, E_{\mathrm{J}}^{\mathrm{a}}=26.4, E_{\mathrm{C}}^{\mathrm{b}}=3.7$, $E_{\mathrm{J}}^{\mathrm{b}}=8.9, \delta E_{\mathrm{J}}=0.17, E_{\mathrm{g}}^{\mathrm{b}}=5$, and $E_{\mathrm{g}}^{\mathrm{a}}=1750$. Using Eqs. (10), these parameters yield $E_{\mathrm{C}}^{\mathrm{si}}=2.5$ and $E_{L}=$ 0.53 ; all energies are in units of $h \mathrm{GHz}$.

[1] Yu. Makhlin, G. Schön, and A. Shnirman, Quantum-State Engineering with Josephson-Junction Devices, Rev. Mod. Phys. 73, 357 (2001).

[2] M.H. Devoret and J. M. Martinis, Implementing Qubits with Superconducting Integrated Circuits, Quantum Inf. Process. 3, 163 (2004).

[3] R. J. Schoelkopf and S. M. Girvin, Wiring Up Quantum Systems, Nature (London) 451, 664 (2008).

[4] J. Clarke and F. K. Wilhelm, Superconducting Quantum Bits, Nature (London) 453, 1031 (2008).

[5] H. Paik, D. I. Schuster, L. S. Bishop, G. Kirchmair, G. Catelani, A.P. Sears, B. R. Johnson, M.J. Reagor, L. Frunzio, L. I. Glazman, S. M. Girvin, M. H. Devoret, and R.J. Schoelkopf, Observation of High Coherence in Josephson Junction Qubits Measured in a ThreeDimensional Circuit QED Architecture, Phys. Rev. Lett. 107, 240501 (2011).

[6] V. Bouchiat, D. Vion, P. Joyez, D. Esteve, and M. H. Devoret, Quantum Coherence with a Single Cooper Pair, Phys. Scr. T76, 165 (1998).

[7] Y. Nakamura, Yu. A. Pashkin, and J.S. Tsai, Coherent Control of Macroscopic Quantum States in a SingleCooper-Pair Box, Nature (London) 398, 786 (1999).

[8] V.E. Manucharyan, J. Koch, L. I. Glazman, and M. H. Devoret, Fluxonium: Single Cooper-Pair Circuit Free of Charge Offsets, Science 326, 113 (2009).

[9] V. E. Manucharyan, N. A. Masluk, A. Kamal, J. Koch, L. I. Glazman, and M.H. Devoret, Evidence for Coherent Quantum Phase Slips across a Josephson Junction Array, Phys. Rev. B 85, 024521 (2012). 
[10] E. Chow, P. Delsing, and D. B. Haviland, Length-Scale Dependence of the Superconductor-to-Insulator Quantum Phase Transition in One Dimension, Phys. Rev. Lett. 81, 204 (1998).

[11] D. B. Haviland, K. Andersson, and P. Ågren, Superconducting and Insulating Behavior in OneDimensional Josephson Junction Arrays, J. Low Temp. Phys. 118, 733 (2000).

[12] Y. Takahide, H. Miyazaki, and Y. Ootuka, Superconductor-Insulator Crossover in Josephson Junction Arrays due to Reduction from Two to One Dimension, Phys. Rev. B 73, 224503 (2006).

[13] I. M. Pop, K. Hasselbach, O. Buisson, W. Guichard, B. Pannetier, and I. Protopopov, Measurement of the CurrentPhase Relation in Josephson Junction Rhombi Chains, Phys. Rev. B 78, 104504 (2008).

[14] I. M. Pop, I. Protopopov, F. Lecocq, Z. Peng, B. Pannetier, O. Buisson, and W. Guichard, Measurement of the Effect of Quantum Phase Slips in a Josephson Junction Chain, Nat. Phys. 6, 589 (2010).

[15] M. T. Bell, I. A. Sadovskyy, L. B. Ioffe, A. Yu. Kitaev, and M.E. Gershenson, Quantum Superinductor with Tunable Nonlinearity, Phys. Rev. Lett. 109, 137003 (2012).

[16] N. A. Masluk, I. M. Pop, A. Kamal, Z. K. Minev, and M.H. Devoret, Microwave Characterization of Josephson Junction Arrays: Implementing a Low Loss Superinductance, Phys. Rev. Lett. 109, 137002 (2012).

[17] R. M. Bradley and S. Doniach, Quantum Fluctuations in Chains of Josephson Junctions, Phys. Rev. B 30, 1138 (1984).

[18] M. Y. Choi, Persistent Current and Voltage in a Ring of Josephson Junctions, Phys. Rev. B 48, 15920 (1993).

[19] Z. Hermon, E. Ben-Jacob, and G. Schön, Charge Solitons in One-Dimensional Arrays of Serially Coupled Josephson Junctions, Phys. Rev. B 54, 1234 (1996).

[20] A. A. Odintsov, One-Dimensional Josephson Arrays as Superlattices for Single Cooper Pairs, Phys. Rev. B 54, 1228 (1996).

[21] L. I. Glazman and A. I. Larkin, New Quantum Phase in a One-Dimensional Josephson Array, Phys. Rev. Lett. 79, 3736 (1997).

[22] M.-S. Choi, J. Yi, M. Y. Choi, J. Choi, and S.-I. Lee, Quantum Phase Transitions in Josephson-Junction Chains, Phys. Rev. B 57, R716 (1998).

[23] K. A. Matveev, A. I. Larkin, and L. I. Glazman, Persistent Current in Superconducting Nanorings, Phys. Rev. Lett. 89, 096802 (2002).

[24] P. Goswami and S. Chakravarty, Dissipation, Topology, and Quantum Phase Transition in a One-Dimensional Josephson Junction Array, Phys. Rev. B 73, 094516 (2006).

[25] J. Homfeld, I. Protopopov, S. Rachel, and A. Shnirman, Charge Solitons and Their Dynamical Mass in OneDimensional Arrays of Josephson Junctions, Phys. Rev. B 83, 064517 (2011).
[26] G. Catelani, R. J. Schoelkopf, M. H. Devoret, and L. I. Glazman, Relaxation and Frequency Shifts Induced by Quasiparticles in Superconducting Qubits, Phys. Rev. B 84, 064517 (2011).

[27] G. Rastelli, I. M. Pop, W. Guichard, and F. W. J. Hekking, Quantum Phase-Slips in Josephson Junction Rings, arXiv:1201.0539.

[28] L. B. Ioffe, M. V. Feigel'man, A. Ioselevich, D. Ivanov, M. Troyer, and G. Blatter, Topologically Protected Quantum Bits Using Josephson Junction Arrays, Nature (London) 415, 503 (2002).

[29] A. Kitaev, Protected Qubit Based on a Superconducting Current Mirror, arXiv:cond-mat/0609441.

[30] S. Gladchenko, D. Olaya, E. Dupont-Ferrier, B. Douçot, L. B. Ioffe, and M.E. Gershenson, Superconducting Nanocircuits for Topologically Protected Qubits, Nat. Phys. 5, 48 (2008).

[31] B. Douçot and L.B. Ioffe, Physical Implementation of Protected Qubits, Rep. Prog. Phys. 75, 072001 (2012).

[32] E. U. Condon and G. H. Shortley, The Theory of Atomic Spectra (Cambridge University Press, Cambridge, England, 1935).

[33] M. H. Devoret, in Quantum Fluctuations, Proceedings of the Les Houches Summer School, Session LXIII (Elsevier, Amsterdam, 1997), Chap 10.

[34] G. Burkard, R. H. Koch, and D. P. DiVincenzo, Multilevel Quantum Description of Decoherence in Superconducting Qubits, Phys. Rev. B 69, 064503 (2004).

[35] F. London, Superfluids (Wiley, New York, 1950), Vol. I, p. 152.

[36] R. Doll and M. Näbauer, Experimental Proof of Magnetic Flux Quantization in a Superconducting Ring, Phys. Rev. Lett. 7, 51 (1961).

[37] B.S. Deaver, Jr. and W. M. Fairbank, Experimental Evidence for Quantized Flux in Superconducting Cylinders, Phys. Rev. Lett. 7, 43 (1961).

[38] D. A. Ivanov, L. B. Ioffe, V. B. Geshkenbein, and G. Blatter analyzed dihedral symmetry for a ring of four identical Josephson junctions with zero or $\pi$ external flux in Interference Effects in Isolated Josephson Junction Arrays with Geometric Symmetries, Phys. Rev. B 65, 024509 (2001). For the system we discuss in this article, discrete rotational symmetry is broken due to the weak junction.

[39] J. Koch, V.E. Manucharyan, M.H. Devoret, and L.I. Glazman, Charging Effects in the Inductively Shunted Josephson Junction, Phys. Rev. Lett. 103, 217004 (2009).

[40] This term was introduced by A. Kitaev; see also Ref. [15].

[41] M. Hamermesh, Group Theory and Its Application to Physical Problems (Dover, New York, 1989).

[42] B. E. Sagan, The Symmetric Group (Springer, New York, 2000). 